\title{
Using near-infrared spectroscopy for characterization of transiting exoplanets
}

\author{
E. Aronson and P. Waldén
}

\author{
Observational Astronomy, Department of Physics and Astronomy, Uppsala University, Box 516, 75120 Uppsala, Sweden \\ e-mail: erik.aronson@physics.uu.se
}

Received 24 April 2014 / Accepted 27 March 2015

\section{ABSTRACT}

\begin{abstract}
Context. We propose a method for observing transiting exoplanets with near-infrared high-resolution spectrometers. Aims. We aim to create a robust data analysis method for recovering atmospheric transmission spectra from transiting exoplanets over a wide wavelength range in the near-infrared.

Methods. By using an inverse method approach, combined with stellar models and telluric transmission spectra, the method recovers the transiting exoplanet's atmospheric transmittance at high precision over a wide wavelength range. We describe our method and have tested it by simulating observations.

Results. This method is capable of recovering transmission spectra of high enough accuracy to identify absorption features from molecules such as $\mathrm{O}_{2}, \mathrm{CH}_{4}, \mathrm{CO}_{2}$, and $\mathrm{H}_{2} \mathrm{O}$. This accuracy is achievable for Jupiter-size exoplanets at $\mathrm{S} / \mathrm{N}$ that can be reached for $8 \mathrm{~m}$ class telescopes using high-resolution spectrometers $(R>20000)$ during a single transit, and for Earth-size planets and super-Earths transiting late $\mathrm{K}$ or $\mathrm{M}$ dwarf stars at $\mathrm{S} / \mathrm{N}$ reachable during observations of less than 10 transits. We also analyse potential error sources to show the robustness of the method.

Conclusions. Detection and characterization of atmospheres of both Jupiter-size planets and smaller rocky planets looks promising using this set-up.
\end{abstract}

Key words. methods: data analysis - techniques: spectroscopic - planets and satellites: atmospheres - infrared: planetary systems

\section{Introduction}

The next big steps in exoplanetary science is to investigate the physical conditions on worlds outside our solar system. One of the most promising methods for this is so-called transit spectroscopy, which makes it possible to determine physical parameters of the exoplanetary atmosphere, most noticeably the chemical composition, but possibly also pressure and temperature profiles, rotation period, global weather patterns, cloud coverage, etc.

From radial velocity measurements and transit light curves, orbital parameters, radius, mass, and mean density of an exoplanet can be calculated (Winn 2010). With this, one can divide exoplanets into categories, such as gas giants, ice giants, and rocky planets. A large number of the discovered exoplanets are categorized as hot-Jupiters, which are gas giants orbiting their stars often at distances closer than Mercury to the Sun. The ubiquity of such objects represents a challenge to our planetary-system formation theories (Boss 1997; Nagasawa et al. 2008; Coleman \& Nelson 2014). Another problem is the discrepancy between mass and radius for many smaller exoplanets, which falls between the rocky and gas planets of the solar system. Several possible solutions have been proposed, such as ocean planets, rocky planets with large extended atmospheres, or metal-poor planetary cores (similar to that of the moon), but the true nature of these worlds remains unclear (Léger et al. 2004; Castan \& Menou 2011; Rogers \& Seager 2010).

Monitoring stellar brightness over long periods of time in search of exoplanet transits (i.e. when the exoplanet moves in front of its host star from our points of view) has proven to be a very successful detection method. Since the first observed transit event (Charbonneau et al. 2000), more than one thousand (November 2014) transiting exoplanets have been discovered. The success of the transit method for exoplanet detection comes mainly from the ability to observe many potential systems simultaneously.

The feasibility of exoatmosphere detection by transit observations has been demonstrated by several authors (Brown 2001; Hubbard et al. 2001; Seager \& Sasselov 2000). During the transit, stellar light can pass directly though the semi-transparent part of the atmosphere. The low contrast ratio between an exoplanetary atmosphere and its host star makes detecting the atmosphere a challenging task. The projection of a planetary atmosphere for a solar-size star covers of the order of $10^{-3}$ of the stellar disk for Jupiter-size planets, and can be as low as $10^{-6}$ for super-Earths and Earth-size planets.

Many successful detections using transit spectroscopy have been made. The two most commonly used methods for obtaining transit spectra are (1) low-resolution spectroscopy or photometric measurements of the transit light curve in filters corresponding to typical broadband absorption features, which was first demonstrated by Jha et al. (2000); and (2) high-resolution narrow-band spectroscopy targeting a specific absorption line (or band of lines), first demonstrated by Charbonneau et al. (2002). The transit curves from the first method are used to calculate the radius of the planet as a function of wavelength. Differences in perceived radius of a planet in different filters are interpreted as the presence of a semi-transparent atmosphere. This method makes it possible to recreate the transmission spectrum over a wide wavelength range, albeit at low spectral resolution. Molecules responsible for the observed absorption features typically can not be identified directly due to low resolution. 
Instead one usually tries to find the best fitting model of the planetary atmosphere based on a library of such models. The second method depends less on models. Here one targets an absorption line of a molecule assumed to be present in the atmosphere of a planet. By measuring the relative intensity dip at wavelengths corresponding to this line and the intensity at wavelengths close to this, the presence of the molecule can be detected. Both these methods are applicable to ground- and space-based observations, although space offers a number of obvious advantages.

One of the best studied hot-Jupiters is HD 209485 b, which is slightly larger than Jupiter $\left(R_{\mathrm{p}}=1.36 R_{\mathrm{Jup}}\right)$ and which orbits a bright $(V$ mag $=7.7)$ solar-like star. HST STIS spectra of this exoplanet during transit has led to the detection of sodium (Charbonneau et al. 2002), atomic hydrogen (Vidal-Madjar et al. 2003; Ballester et al. 2007), oxygen, carbon (Vidal-Madjar et al. 2004), magnesium (Vidal-Madjar et al. 2013), and molecular hydrogen (Lecavelier Des Etangs et al. 2008). Using a new observational strategy (ground-based high-resolution spectroscopy, CRIRES at VLT) and data analysis method, Snellen et al. (2010) detected CO absorption in the exoplanet's atmosphere. Another well-studied hot-Jupiter is HD 189733 b, for which methane and water (Swain et al. 2008) were detected using HST observations, and sodium was detected through high-resolution ground-based observation (HRS at HET; Redfield et al. 2008).

Smaller exoplanets require smaller host stars such that $R_{\text {star }} / R_{\text {planet }}$ favours detection of exoplanetary atmospheres. The atmospheric transmittance of the Neptune-size exoplanet GJ 436 b has been measured, revealing a mostly featureless spectrum in near-infrared (NIR; Pont et al. 2009; Knutson et al. 2014).

The most extensively studied super-Earth is GJ $1214 \mathrm{~b}$ (Bean et al. 2010, 2011; Carter et al. 2011; Croll et al. 2011; Crossfield et al. 2011; Désert et al. 2011; Berta et al. 2012; Murgas et al. 2012; de Mooij et al. 2012, 2013; Colón \& Gaidos 2013; Fraine et al. 2013; Narita et al. 2013; Teske et al. 2013; Wilson et al. 2014; Cáceres et al. 2014; Kreidberg et al. 2014). The small host star (an M dwarf with $R_{\text {star }}=0.21 R_{\odot}$ ) gives the planet a favourable contrast ratio. The combined wavelength coverage from these studies ranges from 0.4 to $5 \mu \mathrm{m}$. Most studies have concluded that GJ 1214 b has a flat and featureless transmission spectrum, usually suggested to be caused either by a waterdominated atmosphere or by high altitude clouds or hazes.

These efforts have shown that with current methods and instruments, it is indeed possible to detect exoplanetary transmission spectra. The strong model dependence of the fitting procedure may, however, introduce uncertainties and degeneracies in the analysis of the atmosphere. The goal of this paper is to present an alternative observational strategy and data analysis that is capable of obtaining exoplanetary atmospheric transmittance over a wide wavelength range with sufficient resolution to identify the sources of spectral features directly from their shapes and placements. From such a spectrum, the chemical composition and physical conditions in the atmospheres could be deduced with less model dependence. Examples in this paper are in NIR due to our interest and involvement in the CRIRES+ upgrade (see Sect. 5), but we foresee no reasons that this could not be applied to other instruments or wavelength domains. We also formulate the instrumental requirements needed to obtain suitable observations for this method.

\section{Method}

Here we describe the data analysis method for ground-based high-resolution transit spectroscopy.

\subsection{Synthetic observations}

To test the data analysis method, we need observations of transiting exoplanets. To have control over the data and to know the accuracy of the reconstructed exoplanet transmission spectrum, we create artificial observations with a hypothetical highresolution spectrograph. This way we have full control over the signal-to-noise ratio $(\mathrm{S} / \mathrm{N})$, the ratio of planetary-to-stellar radius, atmospheric conditions, radial velocity, etc., and thus are able to reliably estimate the requirements for instruments and target selection. The simulated observations are based on several assumptions, as follows.

1. A solid (or cloud-covered) part of the planet blocks stellar light completely in the observed wavelengths, while the atmosphere above absorbs light as a function of wavelength.

2. The exposure times are short enough to ignore the motion of the planet across the stellar disk during a single exposure.

3. The stellar flux spectrum and exoplanet transmission spectrum remain constant during the whole transit.

4. The noise of observations is dominated by photon statistics.

We simulate several short exposures of the transiting system as the planet moves across the stellar disk. A simulated spectrum $\left(\tilde{S}_{n}\right)$ of a star with a transiting exoplanet reaching our detector is computed as

$\tilde{S}_{n}(\lambda, t)=\left[F\left(\lambda, v_{\mathrm{s}}\right)-i_{\mathrm{p}}\left(\lambda, \phi, v_{\mathrm{s}}\right) \frac{R_{\mathrm{p}}^{2}}{R_{\star}^{2}}-i_{\mathrm{a}}\left(\lambda, \phi, v_{\mathrm{s}}\right) P\left(\lambda, v_{\mathrm{s}+\mathrm{p}}\right)\right] \cdot T(\lambda, t)$

$i_{\mathrm{p}}\left(\lambda, \phi, v_{\mathrm{s}}\right)=\int_{A_{\mathrm{p}}(\phi)} I\left(\lambda, \mu, v_{\mathrm{s}}\right) \mathrm{d} \mu$
$i_{\mathrm{a}}\left(\lambda, \phi, v_{\mathrm{s}}\right)=\int_{A_{\mathrm{a}}(\phi)} I\left(\lambda, \mu, v_{\mathrm{s}}\right) \mathrm{d} \mu$
$P(\lambda)=\frac{H(\lambda)^{2}+2 R_{\mathrm{p}} H(\lambda)}{R_{\star}^{2}}$.

The expression in square brackets replaces the contribution of the obscured area on the stellar surface with the stellar light that passes through the exoplanetary atmosphere. Here $F$ is the flux of the star, and $i_{\mathrm{p}}$ and $i_{\mathrm{a}}$ are the specific intensities at the position of the planet or its atmosphere. These are given by integrating the specific intensity $I F$ (characterized by the normalized limb distance $\mu$ ) over the area of the opaque part of the planet $\left(A_{\mathrm{p}}\right)$ or the semi-transparent atmosphere $\left(A_{\mathrm{a}}\right)$. If the planet moves significantly over the stellar disk during an exposure, the integration can be used to represent this movement, integrating over the motion across the stellar disk. $R_{\mathrm{p}}^{2} / R_{\star}^{2}$ is the fraction of the stellar disk that is obscured by the opaque part of the planet.

Our goal is to recover the transmittance of the exoplanet atmosphere, represented by $P$, where $P$ is calculated using the wavelength dependent height of the opaque atmosphere $H$ and is rescaled in units of stellar disk area. This means that $P$ is the fraction of the stellar disk that is blocked by absorption in the atmosphere. Telluric transmittance is given by $T$, and because of temporal variations (represented by $t$ ), we allow $T$ to change with each exposure. Simulations of observed spectra are made for many short exposures, which are represented by subscript $n$. Each exposure is taken during a given point in the orbit of the exoplanet, represented by phase $\phi$, which in turn corresponds to different $\mu$ as the exoplanet moves across the stellar disk during 


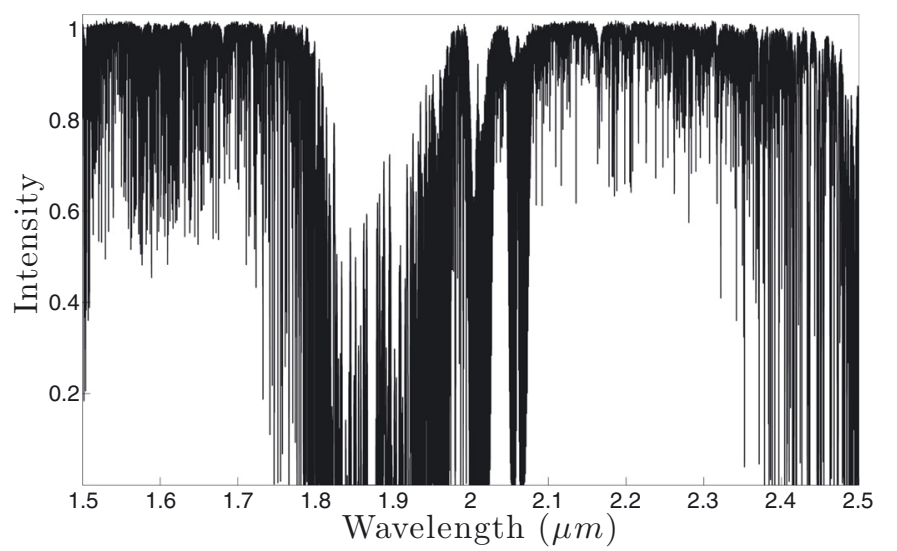

Fig. 1. Simulated observation of a hot-Jupiter transiting solar-like star, at $\mathrm{S} / \mathrm{N} 175$ and spectral resolution $R=100000$. All visible lines have telluric or stellar origins, and the planet's contribution is not visible at this scaling since it is at the level of $10^{-3}$ (for the strongest lines) of stellar contribution.

the transit. The rotation of the Earth (of the order of $0.5 \mathrm{~km} \mathrm{~s}^{-1}$ ), and the star-planet system's velocity relative to Earth will result in a small Doppler shift in the stellar spectrum relative to the instrument, represented by $v_{\mathrm{s}}$. The exoplanet will also move in its orbit during the transit. The radial velocity of a planet in transit is generally small, but for planets orbiting very close to their stars, variations during the transit produces non-negligible red and blue shifts, which is represented by $v_{\mathrm{p}}$. All velocities are given relative to the detector.

The resulting spectrum is convolved with an instrumental profile $(\Gamma)$, which is approximated by a Gaussian with the FWHM given by the spectral resolution of the instrument (which we assume to be constant over the entire wavelength range). We then add white noise to each pixel. The realization of the noise $(N)$ for a certain exposure is given by Poisson distribution, with the width of the distribution given by the inverse of the $S / N$ selected for the data. We have ignored the effect of the blaze on the $\mathrm{S} / \mathrm{N}$ within a spectral order. Finally, the spectrum is continuum normalized $(\eta)$, and with this the full observation is created:

$$
S_{n}(\lambda, t)=\left[\tilde{S}_{n}(\lambda, t) \otimes \Gamma \cdot(1-N(\lambda, \phi))\right] \cdot \eta .
$$

To take the temporal changes in telluric transmittance into account accurately, as well as to use specific intensity corresponding to the different parts of the stellar disk that the planet covers during the transit, it is preferable to use many short exposures (0.5-2 min) of the transit rather than a single long integration. An example of a synthetic observation is given in Fig. 1.

\subsection{Data analysis method}

Once simulated observations are produced, we may use them to reconstruct the transmission spectrum of the exoplanet $(P)$. The straightforward approach would be to rewrite Eqs. (1) and (2) to express $P$ directly:

$$
\begin{aligned}
P(\lambda) \approx & \frac{1}{n} \sum_{n}\left[\frac{-S_{n}\left(\lambda, t,-v_{\mathrm{s}+\mathrm{p}}\right) \cdot v(\phi)}{\left(T\left(\lambda, t,-v_{\mathrm{s}+\mathrm{p}}\right) \cdot i_{\mathrm{a}}\left(\lambda, \phi,-v_{\mathrm{p}}\right)\right) \otimes \Gamma}\right. \\
& \left.+\frac{F\left(\lambda,-v_{\mathrm{p}}\right)-R_{\mathrm{p}}^{2} / R_{\star}^{2} i_{\mathrm{p}}\left(\lambda, \phi,-v_{\mathrm{p}}\right)}{i_{\mathrm{a}}\left(\lambda, \phi,-v_{\mathrm{p}}\right)} \otimes \Gamma\right] .
\end{aligned}
$$

Summation is carried out over many short exposures taken during the transit and then divided by the total number of exposures. Before adding spectra, all spectra are shifted to the reference frame of the exoplanet. Solving Eq. (3), however, fails to recover the exoplanet spectrum since the resulting reconstructed planetary spectrum is swamped by noise amplified in wavelength regions where the denominator (usually telluric transmittance) is small. Rebinning the data to a lower resolution will not help because we will lose the information available between the telluric lines.

An alternative approach is to formulate and solve an inverse problem. Instead of using the expression for $P$ explicitly, we formulate an optimization problem, where we are searching for the function $P$ that minimizes the expression below:

$$
\begin{aligned}
\Phi \equiv & \sum_{n \lambda}\left\{P\left(\lambda, v_{0}\right) \cdot\left[T\left(\lambda, t,-v_{\mathrm{s}+\mathrm{p}}\right) \cdot i_{\mathrm{a}}\left(\lambda, \phi,-v_{\mathrm{p}}\right)\right] \otimes \Gamma\right. \\
& +S_{n}\left(\lambda, t,-v_{\mathrm{s}+\mathrm{p}}\right) \cdot v(t)-\left[F\left(\lambda,-v_{\mathrm{p}}\right) \cdot T\left(\lambda, t,-v_{\mathrm{s}+\mathrm{p}}\right)\right. \\
& \left.\left.+R_{\mathrm{p}}^{2} / R_{\star}^{2} i_{\mathrm{p}}\left(\lambda, \phi,-v_{\mathrm{p}}\right) \cdot T\left(\lambda, t,-v_{\mathrm{s}+\mathrm{p}}\right)\right] \otimes \Gamma\right\}^{2}=\min .
\end{aligned}
$$

This is essentially a rewritten version of Eqs. (1) and (2). To be able to combine spectra, we need all spectra to be on the same wavelength grid. Observations $(S)$, telluric transmittance $(T)$, stellar flux $(F)$, and specific intensity $(i)$ are shifted and interpolated onto the rest frame of the exoplanet. These changes in the wavelength grid are represented by $v_{\mathrm{s}}$ and $v_{\mathrm{p}}$ for stellar radial velocity relative to observer $\left(v_{\mathrm{s}}\right)$ and exoplanet radial orbital velocity relative to the star $\left(v_{\mathrm{p}}\right)$. Exoplanet rest frame is used for establishing the wavelength vector, and we assume that $P$ is constant during the transit. We also assume that the observed spectra is continuum-normalized, but a perfect normalization will differ for the spectra in and outside the transit, accounting for the change in stellar flux. This problem should be addressed from two sides. On the data reduction side, we can use a robust and reproducible algorithm. We have developed such a tool and tested it with a number of fibre-fed and slit spectrographs, including ESO HARPS and UVES and the Keck HIRES. The main idea is to observe the solar spectrum (e.g. reflected from an asteroid) before and after the science exposures keeping instrument configuration. Sensitivity curves derived from the solar spectrum are then used to normalize the science exposures. In the absence of large seeing variations, this techniques provides precise continuum normalization at the level of $10^{-3}$. In addition, we introduce the brightness correction parameter $v$ on the modelling side. This parameter primarily depends on stellar limb darkening and exoplanet radius. The optimal value of $v$ is found by calculating the residuals ( $r$ in Eq. (5)) between observations multiplied with $v$ and continuum normalized synthetic observations in a given phase. We search for the value of $v$ that minimizes the cross-correlation of residual with stellar spectra and residual with telluric spectra (see Eq. (6)):

$$
\begin{aligned}
& r(v, \lambda, t)=\left\{v(t) \cdot S_{n}\left(\lambda, t,-v_{\mathrm{s}}\right)\right. \\
&\left.-\left[\left(F(\lambda)-i_{\mathrm{p}}(\lambda, \phi) \cdot R_{\mathrm{p}}^{2} / R_{\star}^{2}\right) \cdot T\left(\lambda, t,-v_{\mathrm{s}}\right)\right] \bigotimes \Gamma\right\} \\
& \times 1 /\left(i_{\mathrm{a}}(\lambda, \phi) \bigotimes \Gamma\right) \\
& {[r(v) \star F] \cdot[r(v) \star T]=\min . }
\end{aligned}
$$


There are many ways to formulate an inverse problem for $P$. The form given by Eq. (4) has the advantage of avoiding division by quantities that may turn to zero (telluric transmittance) and of differences between subtracted terms not being orders of magnitude. With this set-up, the noise may still introduce large fluctuations around the mean value, which now can be controlled. This is done by selecting weights (see below) along the wavelength grid and adding a regularization term $(R)$, which controls the balance between the smoothness of the solution and the fit to the observation. Now the minimization problem can be written as

$$
\begin{aligned}
& \Omega \equiv \Phi+\Lambda \cdot R=\min \\
& R=\sum_{\lambda}\left(\frac{\mathrm{d} P(\lambda)}{\mathrm{d} \lambda}\right)^{2}
\end{aligned}
$$

The minimum of $\Omega$ is obtained by requiring the derivatives of $\Omega$ to be zero and by linearizing the equation for $R$. Then, $P$ is given by a system of linear equations with a tridiagonal matrix, which is fast and easy to solve. In this exact formulation of Eq. (4), the telluric absorption acts as a weight; in places with strong absorption $(T \approx 0)$, the regularization dominates, keeping the oscillations of the solution under control across the affected intervals. The constant $\Lambda$ is a free parameter, which balances the relative importance of regularization. For a given dataset, a value of $\Lambda$ must be chosen and possibly be significantly affecting the recovered solution. In our tests of the method, we can compare the obtained solution to the original "true" exoplanet transmission spectrum (what we put into simulated observations). By searching for the $\Lambda$ that gives the smallest least squares difference between these two, we can find an optimal $\Lambda$. When applying this method to the real observations (with the exoplanet spectrum being unknown), the optimal value of $\Lambda$ can be derived in similar numerical experiments using the correct parameters of the system (size of planet to star, duration of transit, instrumental parameters, estimated $\mathrm{S} / \mathrm{N}$ ). The regularization imposes extra smoothness on the solution, but while this dumps numerical noise, it also reduces spectral resolution of the recovered spectrum in places of high telluric absorption.

An additional advantage of this approach to recovering the exoplanetary spectrum is that one can combine observations of several different transits. As long as each single exposure is combined with corresponding telluric and stellar spectra within the summation in Eq. (4), adding exposures from several transits is possible without any additional complications. For this we assume that the exoplanet transmittance does not change from one transit to the next.

In this set-up of the inverse problem, stellar flux spectrum and specific intensity spectra at each $\mu$ point, telluric transmittance and relative areas of star and planet are all needed. How to achieve this is covered in Sect. 4, but for now we assume that all these data are available with sufficient accuracy.

\section{Application}

Now we proceed with testing the proposed method and estimating requirements for both the observed exoplanet system and the instrument.

\subsection{Data sources}

For simulating observations and extracting the exoplanet transmission spectrum, three components are needed, stellar spectra (flux and specific intensity), exoplanet atmospheric
Table 1. Stellar parameters of the host stars used for simulating transit observations.

\begin{tabular}{lll}
\hline \hline & Solar-like & M dwarf \\
\hline Spectral type & G2 & M5 \\
$T_{\text {eff }}(\mathrm{K})$ & 5777 & 3100 \\
$\log (g)(\mathrm{cgs})$ & 4.44 & 5.0 \\
$R / R_{\odot}$ & 1.0 & 0.2 \\
\hline
\end{tabular}

transmission spectrum, and telluric transmittance. For the tests below, the high-resolution flux and specific intensity spectra of the stars were computed with the stellar atmosphere modelling code MARCS (Gustafsson et al. 2008). One-dimensional hydrostatic MARCS models are based on the most advanced atomic and molecular opacities, and they are extensively tested against real data for the Sun, cool dwarfs, sub-giants, and giants. The quality of the stellar model atmospheres was assessed using spectral synthesis, which also gives the estimate of the quality and completeness of the line lists. In the case of the Sun, the comparison was also done for a wavelength-dependent limb darkening. For simulations of observations and for the dataanalysis method, we need the intensity corresponding to the area of the star occulted by the planet or its atmosphere. This is obtained by integrating the specific intensity over the area covered by the planet or by the semi-transparent atmosphere. Variations in specific intensity across the projection of the planetary disk are much less than 1\% (ignoring the presence of active regions), and the integration over the planetary disk can be replaced safely by the cental value. However, in a some cases this is important to include, such as when observing large planets transiting small stars (especially close to the limb of the star), when only part of the planet covers the stellar disk (being able to include these cases enables us to acquire a few additional exposures at the beginning and end of the transit) or when the exposure time is long and the planet moves across the stellar disk during the exposure. In our tests we have focused on two different cases: a Jupiter-size planet in front of a solar-like star and a super-Earth in front of an M dwarf. Flux and intensity spectra were computed for two stars with parameters according to Table 1 .

The telluric transmittance was calculated using the atmospheric modelling code LinePak ${ }^{1}$ (Gordley et al. 1994), which provides calculations of transmission spectra of the telluric atmosphere under given conditions. The atmospheric model used in LinePak for integrating the transmission spectrum are the US Standard Atmosphere, which provides pressure and temperature profiles, as well as partial pressures for various gases. In particular, the molecules dominating the NIR absorption such as $\mathrm{O}_{2}, \mathrm{O}_{3}, \mathrm{H}_{2} \mathrm{O}, \mathrm{CO}_{2}, \mathrm{CH}_{4}$, and $\mathrm{HNO}_{3}$ are all included in the calculations. The line lists used for calculating the absorption bands of these molecules were taken from HITRAN (Rothman et al. 2009). To represent the typical viewing conditions for astronomical instruments, the altitude was set to $2.6 \mathrm{~km}$ and the water vapour concentration reduced to $20 \%$ of the default concentration in the US Standard Atmosphere where PWV $=14.3 \mathrm{~mm}$. Observations are then synthesized with an airmass varying from 1.0 to 1.2 during a single transit, to represent the star moving downwards from zenith during three hours of observations.

The projected exoplanetary atmosphere is characterize by its height. We define atmosphere height (often referred to as eclipsing height) as the additional radius that a completely opaque disk would have. This disk blocks the same amount of stellar light as

Implemented on the website http://spectralcalc.com 

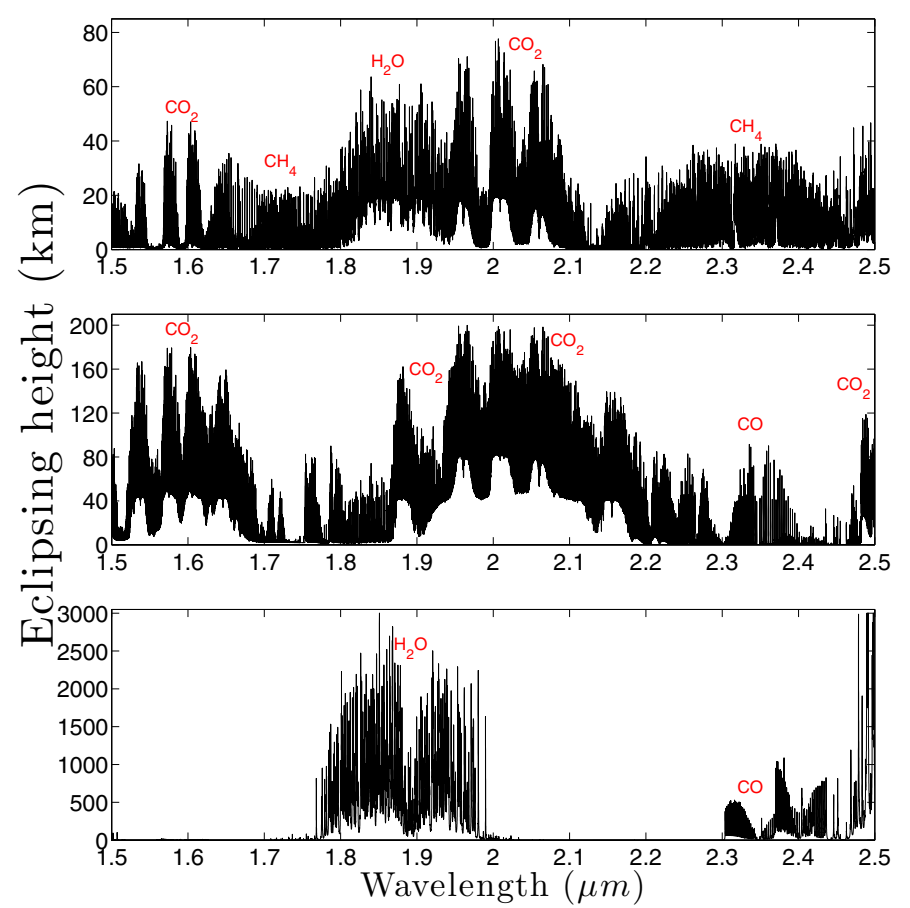

Fig. 2. Eclipsing height of model exoplanet atmospheres as function of wavelength in the $H$ and $K$ NIR-bands. Upper panel: super-Earth with Earth-like composition. Middle panel: super-Earth with Venus-like composition. Bottom panel: hot-Jupiter.

the planet with a semitransparent atmosphere. At wavelengths where the atmosphere is mostly opaque, a larger opaque disk is needed to block the same amount of light, and thus the eclipsing height of the atmosphere is larger at wavelengths where the atmosphere absorbs more light. For the Earth viewed in NIR, the radius would vary between $6385 \mathrm{~km}$ (in wavelength regions with no absorption) and $6470 \mathrm{~km}$ (in wavelength regions with much absorption), making the eclipsing atmosphere height between 0 and $85 \mathrm{~km}$.

Transmission spectra for super-Earth atmospheres (Fig. 2) were created using the same model as the telluric transmission spectrum (LinePak), but increasing the impact parameter of the rays up to $100 \mathrm{~km}$ above the surface. We used two different atmospheric models: one with temperature, pressure, and composition identical to Earth and one more Venus-like, with $\mathrm{CH}_{4}$ and $\mathrm{H}_{2} \mathrm{O}$ replaced by $\mathrm{CO}$ and $\mathrm{CO}_{2}$. Using an exo-atmosphere with a different composition than the Earth's enabled us to test that the data analysis method produces artificial features originating in the telluric atmosphere, while an Earth-like atmosphere enabled us to test whether we can reproduce spectral features that also exist in the telluric atmosphere. Super-Earths typically have surface gravity within $0.9-1.1$ of the Earth's (exoplanet orbit database Wright et al. 2011), which makes the atmospheric scale height for super-Earths with the same atmospheric temperature and mean molecular weight as the Earth or very similar to the Earth's $(8.5 \mathrm{~km})$. Therefore we used the atmosphere height directly from the telluric model. For the Venus-like super-Earth, we wanted to test a more extended atmosphere. If the planet is hotter, the scale height will increase. We used a scale height that is twice as large as for the Earth. On top of this, the $\mathrm{CO}_{2}$ in the atmosphere absorbs light very efficiently, making the eclipsing atmosphere height of the Venus-like planet almost $200 \mathrm{~km}$. For planetary radius, we used $2.5 R_{\oplus}$ in both cases.
Hot-Jupiter model spectra (Fig. 2) were derived in a similar manner, computing the optical length of the rays at different distances from planet centre. The total transmittance was then evaluated using the projected areas of the corresponding concentric rings. The atmospheric structure was taken from Miguel \& Kaltenegger (2014). Opacity contributors used in this model include $\mathrm{H}_{2}, \mathrm{CH}_{4}, \mathrm{CO}, \mathrm{N}_{2}, \mathrm{NH}_{3}, \mathrm{H}_{2} \mathrm{O}, \mathrm{PH}_{3}, \mathrm{H}_{2} \mathrm{~S}, \mathrm{TiO}, \mathrm{VO}, \mathrm{CaH}$, $\mathrm{MgH}, \mathrm{FeH}, \mathrm{CrH}, \mathrm{SiO}, \mathrm{CaOH}$, and $\mathrm{TiH}$. (We do not include any clouds or hazes.) The model atmosphere height is $5600 \mathrm{~km}$ (scale height $500 \mathrm{~km}$ ) on top of a completely opaque core with radius $90000 \mathrm{~km}$. This model planet is slightly larger than the average hot-Jupiter. We scale the radius and eclipsing atmosphere height to match a Jupiter-size planet (radius $69900 \mathrm{~km}$ ). Roughly four-fifths of currently known hot-Jupiters are Jupiter size or larger, making this a conservative estimate for the size of a hot-Jupiter.

\subsection{Simulation set-up}

We simulated observations of three cases (hot-Jupiter transiting solar-like star and Venus and Earth-like rocky planets transiting M dwarfs) during transit and applied the data-analysis method to these "observations". Simulations were performed with a hypothetical state of the art high-resolution spectrograph with spectral resolution $R=100000$ and simultaneous wavelength coverage $1.5-2.5 \mu \mathrm{m}$. The $\mathrm{S} / \mathrm{N}$ of simulated observations is based on estimates of what modern instruments at $8 \mathrm{~m}$ class telescopes will be able to achieve during $75 \mathrm{~s}$ exposures of the two stars. For a solar-like star $(K \operatorname{mag} 8) S / N \approx 150$ and M-dwarf ( $K \operatorname{mag} 9$ ) $S / N \approx 90$ per resolution element. The $\mathrm{S} / \mathrm{N}$ is estimated to be at the centre of the $K$-band. For simplicity we assumed that the $\mathrm{S} / \mathrm{N}$ is constant throughout the observed spectral range and ignored readout and sky noise (sky emission discussed in Sect. 4.2). If these noise sources are significant, the integration time should be adjusted to reach desirable $\mathrm{S} / \mathrm{N}$ levels.

The duration of a transit is an important parameter when estimating the capabilities of the data analysis method, since it directly limits the total observing time. Transit durations range from slightly below one to many hours, approaching a whole day in extreme cases. The correlation between the size of the star and transit duration is not straightforward. Planets transiting larger stars cross a larger stellar disk, but at the same time the stars are more massive, meaning higher orbital velocities. On top of this, the semi major axis (and to a lesser degree the inclination and eccentricity of the orbit) affects the transit duration. Looking at statistics of transit durations from the Exoplanet Orbit Database (Wright et al. 2011), we find longer average transit duration for planets orbiting larger stars (see Fig. 3). For hot-Jupiters transiting solar-like stars (here taken as planets with $R_{\mathrm{p}}>0.5 R_{\text {Jup }}$, $a<0.3$ au and orbiting stars with $0.8 R_{\odot}<R_{\star}>1.2 R_{\odot}$ ), the median transit duration is $165 \mathrm{~min}$. We use this as the transit duration for the hot-Jupiter case.

For the Earth-like super-Earth, we place this planet in the habitable zone (the distance from the star at which liquid water is possible). The inner and outer edges of the habitable zone depend on many things, such as the planet's surface gravity, atmospheric pressure, and greenhouse effects. The eccentricity of the orbit and the rotation of the planet complicate this further. To get rough estimates of the habitable zone, we use the orbital distance of the Earth and scale it with inverse square of stellar luminosity, and this distance is then converted to a transit duration (assuming inclination $90^{\circ}$. For an $\mathrm{M}$ dwarf this would give a transit duration of $90 \mathrm{~min}$. For the Venus-like super-Earth, we use the same method and scale the semi-major axis to match the 


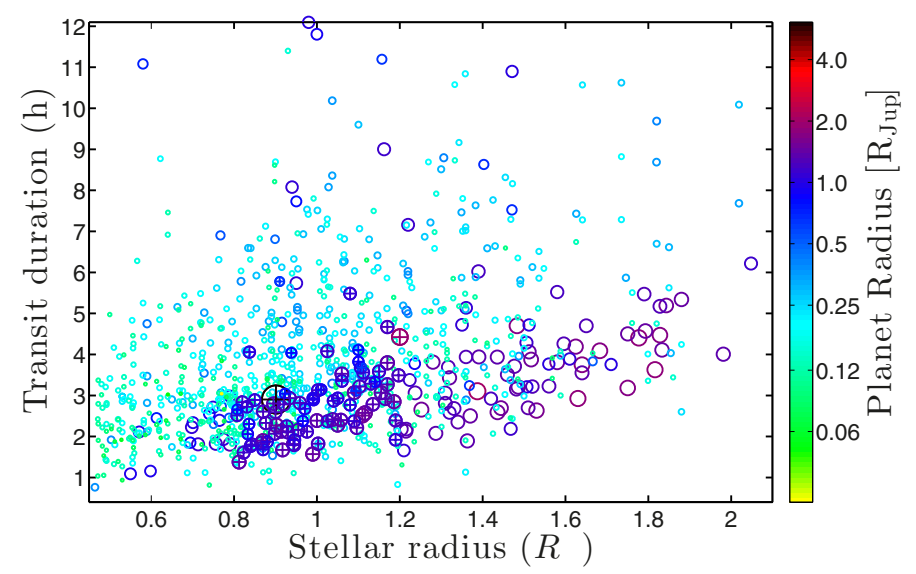

Fig. 3. Transit duration versus stellar radius for 4500 Kepler objects. Colour coding and size of symbols indicate the planetary radius. The $\oplus$ symbols denote hot-Jupiters orbiting solar-like stars.

semi-major axis of Venus, giving us a transit duration of $75 \mathrm{~min}$. See Table 2 for details on orbital parameters.

For each case we show four spectra in the same panel (Fig. 4). The solution of the inverse problem, corresponding to the recovered exoplanet transmission spectrum, is shown in red. To show how accurate the recovery is, the original transmission spectrum of the exoplanetary atmosphere is shown in black. Along with this we over plot (dashed grey line) the input exoplanet spectrum with the same amount of weighted regularization as the recovered solution. These spectra are represented by their atmospheric height (defined as in Sect. 3.1), with the axis on left-hand side of each figure. We also include the telluric transmission in greyish blue to indicate the parts of the wavelength regime where the telluric transmittance, hence the signal, is very low. This is represented by the transmittance axis on the right-hand side of each figure.

Results from the tests are shown in Fig. 4. For the hot-Jupiter ( $\left.R=1 R_{\text {Jup }}\right)$ transiting a solar-like star, the strongest recovered absorption bands coincide with the major absorption bands in the input spectrum. In the tests with a super-Earth with Earthlike transmittance, we had to combine observations from four transits (corresponding to a total of $6 \mathrm{~h}$ of observations) in order to recover the major $\mathrm{H}_{2} \mathrm{O}, \mathrm{CO}_{2}$, and $\mathrm{CH}_{4}$-bands, and even with observations from four transits, the results are noisy and contain a few spurious features. The Venus-like exoplanet is more advantageous than the Earth-like case mainly because of its larger atmosphere, and we manage to recover the major absorption bands during a single 75 -min-long transit.

\section{Requirements}

Owing to the very small fraction of the stellar disk that is blocked by the exoplanet atmosphere, recovering exoplanetary transmission spectra puts heavy requirements on observations, input data, and the targeted planetary system. Based on simulations of varying input parameter, we tried to estimate requirements for transit spectroscopy using this data analysis method. Unless noted otherwise, all tests in this section are done with the same planet and star parameters as the Venus-like super-Earth case above, see Table 2.
Table 2. Physical properties of star-exoplanet systems.

\begin{tabular}{llll}
\hline \hline & $\begin{array}{l}\text { Hot. } \\
\text { Jupiter }\end{array}$ & $\begin{array}{l}\text { Earth- } \\
\text { like }\end{array}$ & $\begin{array}{l}\text { Venus- } \\
\text { like }\end{array}$ \\
\hline Semi-major axis $(\mathrm{au})$ & 0.054 & 0.064 & 0.046 \\
Inclination $i$ & $90^{\circ}$ & $90^{\circ}$ & $90^{\circ}$ \\
Orbital period (days) & 4.5 & 13.3 & 8.1 \\
Transit duration (min) & 165 & 90 & 75 \\
$R_{\mathrm{p}}^{2} / R_{\star}^{2}$ & 0.010 & 0.013 & 0.013 \\
System velocity $v_{\mathrm{s}}\left(\mathrm{km} \mathrm{s}^{-1}\right)$ & 15 & 15 & 15 \\
Orbital velocity $K_{\mathrm{p}}\left(\mathrm{km} \mathrm{s}^{-1}\right)$ & 130 & 52 & 62 \\
Velocity semi-amplitude $K_{\star}(\mathrm{m} / \mathrm{s})$ & 124 & 6.5 & 7.0 \\
$K$ mag & 8.0 & 9.0 & 9.0 \\
\hline
\end{tabular}
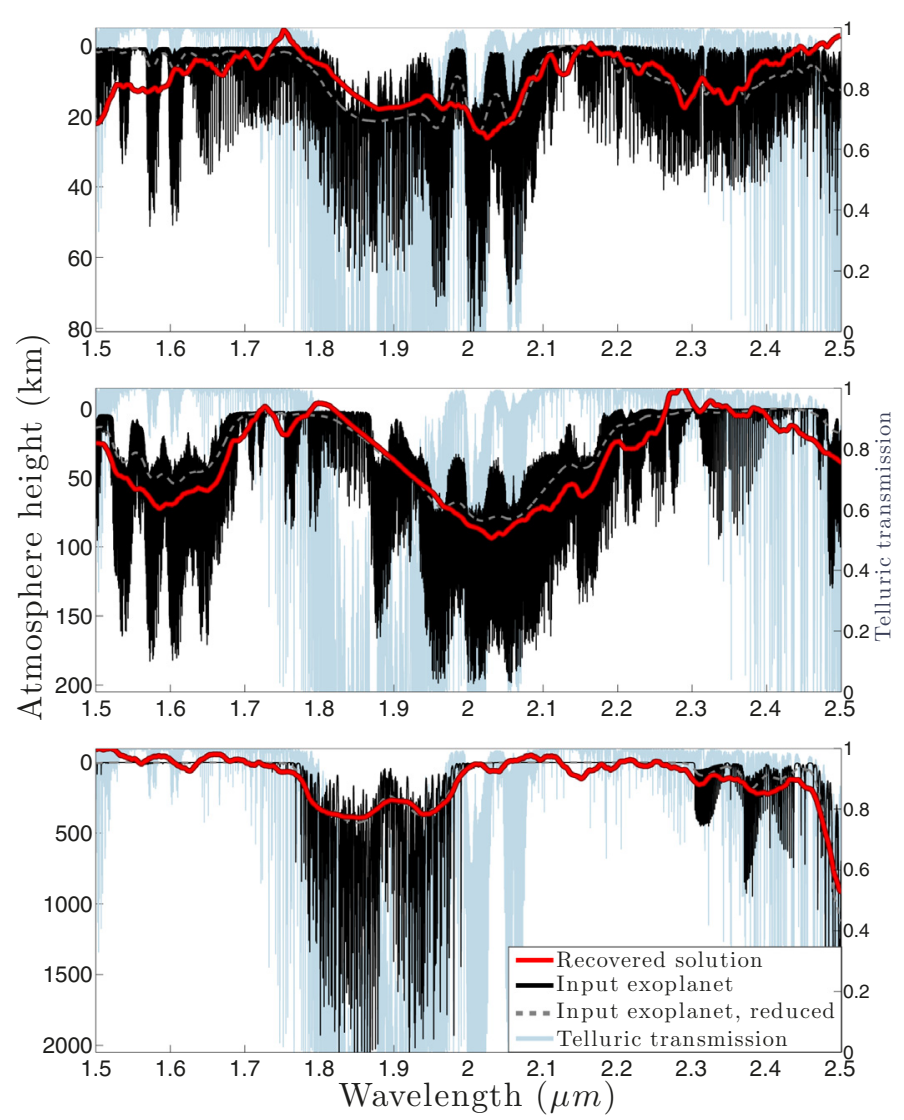

Fig. 4. Recovered exoplanetary transmission spectra from simulated transit observations. Upper panel: Earth-like super-Earth transiting M5 star, 4 transits. Middle panel: Venus-like super-Earth transiting M5 star, 1 transit. Lower panel: hot-Jupiter transiting G2 star, 1 transit.

\subsection{Observational requirements}

The two most important characteristics of observations, and therefore the instrument, are (1) spectral resolution; and (2) wavelength coverage.

1. Spectral resolution: the resolution of the spectrograph is a vital aspect of the instrument. With high resolution, we are able to see between telluric lines and detect contribution from the exoplanet that might not be observable if using lower resolution. We can use this to see molecular species present in both the telluric and the exoplanetary atmosphere, since there will be a small radial velocity difference between the telluric atmosphere and the exoplanet. This will shift the exoplanetary lines relative to the telluric lines, and this difference is detectable with high resolution. With a varying radial velocity 

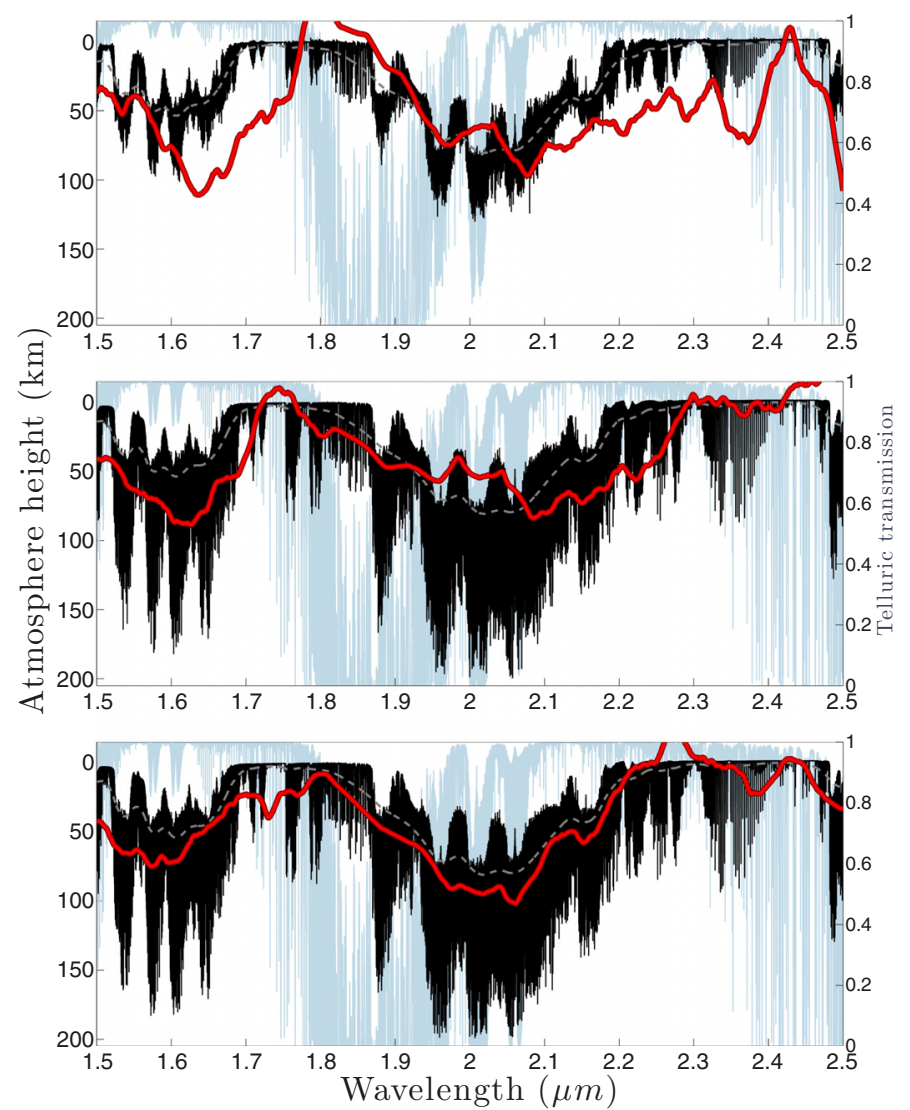

Fig. 5. Recovered exoplanetary transmission spectra from simulated transit observations of hot super-Earth transiting M5 star. We simulate observations using instruments with different spectral resolution to show the importance of high spectral resolution. Upper panel: spectral resolution: $R=10000$. Middle panel: spectral resolution: $R=50000$. Lower panel: spectral resolution: $R=100000$. Colour coding is the same as in Fig. 4.

difference during a transit, we will also be able to scan for exoplanetary lines superpositioned on telluric features, thanks to the rotation of the Earth and the orbit of the exoplanet.

To show the importance of high resolution, we simulated observations with spectral resolution of 10000, 50000 and 100000 and applied the data-analysis method. Results of these tests are shown in Fig. 5. Recovering the exoplanet spectrum is possible at resolutions less than 100000 , but better spectral resolution relaxes the required $\mathrm{S} / \mathrm{N}$. At resolution $R=50000$ recovering exoplanet spectrum in regions with strong telluric absorption is difficult. At spectral resolution below 50000 , telluric and exoatmospheric lines blend, and the recovery of exoplanet spectrum deteriorates. It is no longer possible to differentiate spurious features from true absorption bands.

There are two factors that affect the resolution of the recovered exoplanet spectrum, the instrumental resolution and the regularization parameter. If data quality is very high (i.e. high $\mathrm{S} / \mathrm{N}$ and/or many combined transits), one can afford to reduce regularization and still recover exoplanet transmission spectrum with high accuracy. In the most favourable cases, when combining many transits of bright star with a transiting hot-Jupiter, we are able to reconstruct individual lines. To illustrate the effect of high resolution, we simulated observations from 15 transits of the same Jupiter-size exoplanet and solar-like star as used previously. We do this with spectral resolutions of 100000,50000 , and 10000 . We show a small region of the reconstructed exoplanetary spectrum where we reconstruct individual lines (see Fig. 6). When using spectral resolution of 100000 and 50000 the spectral features ( $\mathrm{CO}$ lines in this case) are reconstructed. When using a resolution of 10000 , the results are significantly worse, and it becomes hard to distinguish between real and spurious features. While the recovered solution appears to have the same spectral resolution (owing to similar amounts of regularization), the difference in the accuracy of the reconstruction depends only here on the resolution of the spectrograph (and thus on the ability to differentiate exoplanetary contribution from telluric lines).

Since the telluric transmittance in NIR contains many spectral features, it is critical to know the telluric atmosphere to high accuracy. In Sect. 4.2 we describe why high resolution can be useful when obtaining telluric transmission spectra.

2. The simultaneous wavelength coverage of the spectrograph is another important factor. Being able to detect a continuum and absorption features simultaneously enables us to correct for errors in planetary radius or estimated relative brightness decrease, which will shift the recovered spectrum along the eclipsing height axis. At least $0.1 \mu \mathrm{m}$ is needed to target the most favourable bands. With very high $\mathrm{S} / \mathrm{N}$, detecting a continuum is possible even in narrow bands (such as in Fig. 6). Being able to cover a significantly wider wavelength range than $0.1 \mu \mathrm{m}$ in a single exposure is very valuable. Observing time is limited by the transit duration, and wide wavelength coverage makes it possible to search for several species simultaneously, as well as to identify what species is responsible for an absorbing region from the shape and component spacing on the feature.

Broader spectral coverage also helps with the continuum normalization of observations. The quality of this step in stellar spectroscopy depends very much on the spectral type of the target and the spectral domain. For cooler stars, molecular bands can prevent proper normalization based solely on the science spectrum. An alternative approach is to measure relative monochromatic throughput of telescope + spectrometer using a well known spectrum. We have tested this techniques with the solar reference spectrum in the optical and NIR using the observations of Vesta and Ganymede taken with the HARPS (ESO La Silla) and the HIRES (Keck) spectrometers. Previous knowledge of the result provided the throughput curves, thereby correcting all small-scale instrumental and detector inhomogeneities to better than $0.5 \%$. Then when applied to the science target, the remaining trend is only due to the difference in spectral type and could be easily adjusted for.

We have chosen to focus on the NIR wavelength regime in this paper. The main benefit to this is the numerous molecular absorption bands located there. In hot-Jupiter atmospheres, we expect to find species, such as $\mathrm{H}_{2} \mathrm{O}, \mathrm{CO}_{2}, \mathrm{CH}_{4}$, $\mathrm{CO}, \mathrm{H}, \mathrm{H}_{2}, \mathrm{O}$, and $\mathrm{OH}$ (Miguel \& Kaltenegger 2014). The expected chemical composition of super-Earths is more uncertain. Extrapolating from the atmospheres of rocky planets in out own solar system indicates that they are likely to be very diverse. The $H, K, L$, and $M$-bands in NIR contain many molecular absorption bands from important molecules, such as $\mathrm{O}_{3}, \mathrm{CO}, \mathrm{CO}_{2}, \mathrm{SO}_{2}, \mathrm{CH}_{4}$, and $\mathrm{NH}_{3}$. In wavelengths longer than $2.5 \mu \mathrm{m}$, sky emission becomes significant, making ground-based observations less competitive with space observations. At shorter wavelengths, sky emission is less significant. In Sect. 4.2 we propose dealing with sky emission 

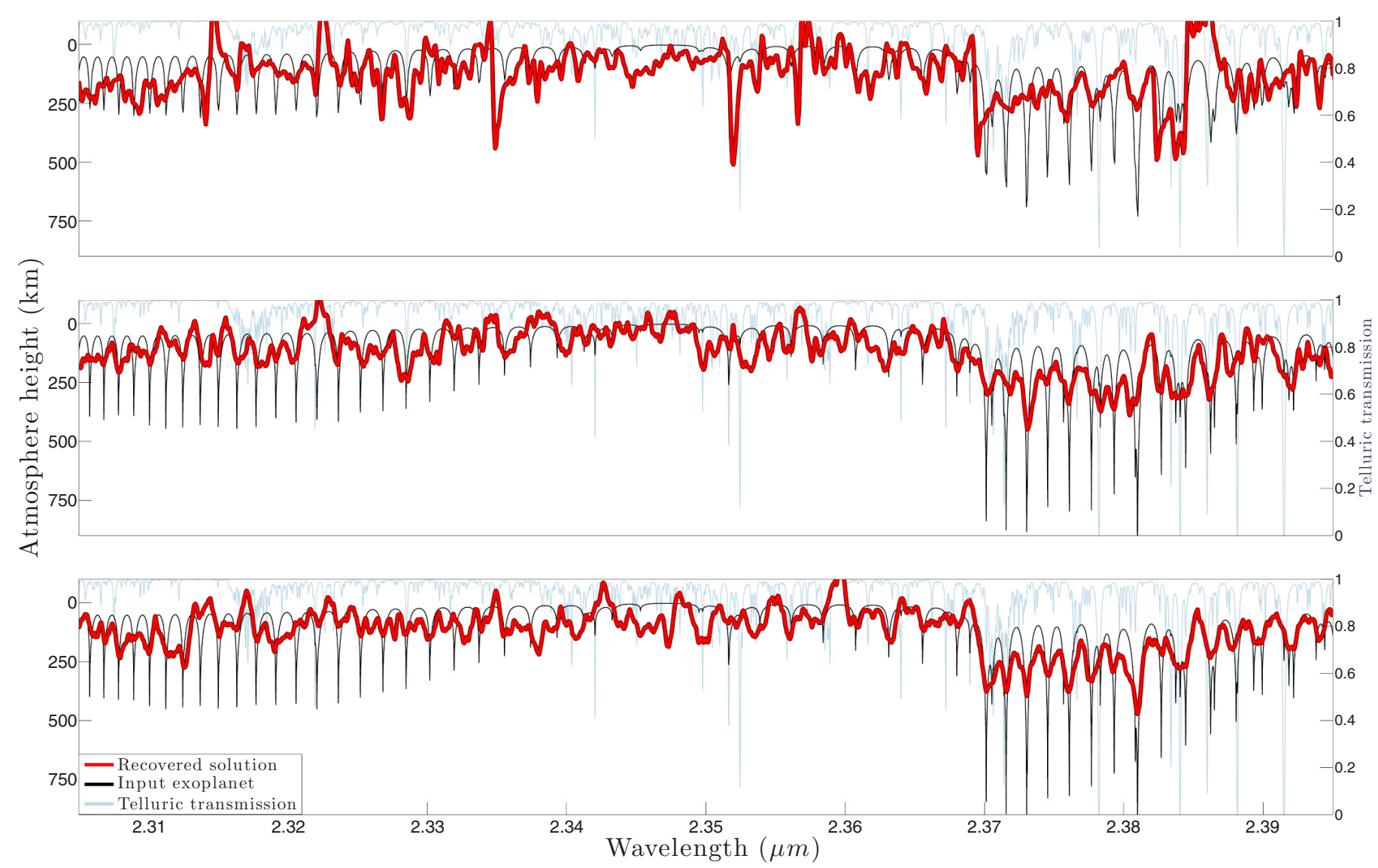

Fig. 6. Recovered exoplanetary transmission spectra from simulated transit observations of a hot-Jupiter transiting G2 star, combing observations of 15 transits. We demonstrate the reconstruction of individual absorption lines made possible by high $\mathrm{S} / \mathrm{N}$ and spectral resolution. Upper panel: spectral resolution: $R=10$ 000. Middle panel: spectral resolution: $R=50$ 000. Lower panel: spectral resolution: $R=100000$.

using spectral synthesis. The $\mathrm{S} / \mathrm{N}$ required to recover the exoplanet transmission spectrum is high, so using wavelengths longer than $3 \mu \mathrm{m}$ will be problematic for all but the largest planets and brightest stars. We are therefore limited to the $H$ and $K$-bands $(1.5-1.8 \mu \mathrm{m}$ and $1.9-2.5 \mu \mathrm{m})$, where we have strong absorption from $\mathrm{CO}_{2}, \mathrm{CO}, \mathrm{CH}_{4}, \mathrm{H}_{2} \mathrm{O}, \mathrm{CH}_{4}$, and $\mathrm{NH}_{3}$. Observations in the $J$-band $(1.1-1.4 \mu \mathrm{m})$ can also be valuable due to the strong $\mathrm{O}_{2}$ absorption band around $1.27 \mu \mathrm{m}$, but unfortunately very few other molecules have absorption bands in this region.

\subsection{Model requirements}

To test the method's robustness, we examined how sensitive our method is to the accuracy of measurements and model spectra. We need telluric transmission spectra separately from the stellar contribution. Observations consists of several short exposures of the system as the planet moves across the stellar disk, and for each of these exposures, the matching telluric transmittance is needed. This must be very accurate and include changes in the telluric atmosphere during the transit. This could be due to changes in airmass as the star moves across the sky or it could be due to changes in the relative abundances of specific species (most notably water vapour).

Such changes will affect line strength and saturation, and small errors in the telluric transmittance used in the data analysis can introduce spurious features in the recovered exoplanetary spectrum. For this reason we do not recommend using preand post-transit observations of telluric standards for obtaining telluric transmittance, since it will be insensitive to changes in the atmosphere during the transit. Dedicating time observing a telluric standard at regular intervals during the transit is not recommended either, because the standard must lie close to the target where small changes in telluric transmittance will affect results.

Since a transit event is limited in time, we want to avoid spending time during the transit not observing the system itself. A more promising method of obtaining the telluric transmittance is synthetic spectra fitted to observations. Several tools for synthesizing high-precision telluric transmission spectra exist, are under development (Seifahrt et al. 2010; Kausch et al. 2014), and can currently reproduce telluric absorption to an accuracy better than methods using telluric standard stars. Ideally such spectral synthesizing includes sky emission to reduce the need for nodding, thus maximizing the time spent observing the transiting planet. In spectral regions where sky emission is significant, nodding might be required in order to produce a telluric model with high enough accuracy. In such cases observation time should take this into account, in our estimations we assume sky emission is either negligible or can be modelled with high accuracy.

To minimize the risk of including contributions from the exoplanet when fitting telluric transmittance to observations, we need high enough spectral resolution to resolve the Doppler shift due to differences in radial velocity between telluric and exoplanet atmosphere. Observations will originally be in the rest frame of the detector. To avoid introducing additional errors, telluric model spectra (and stellar models) should be fitted to observations at this stage, before they are shifted and interpolated onto 

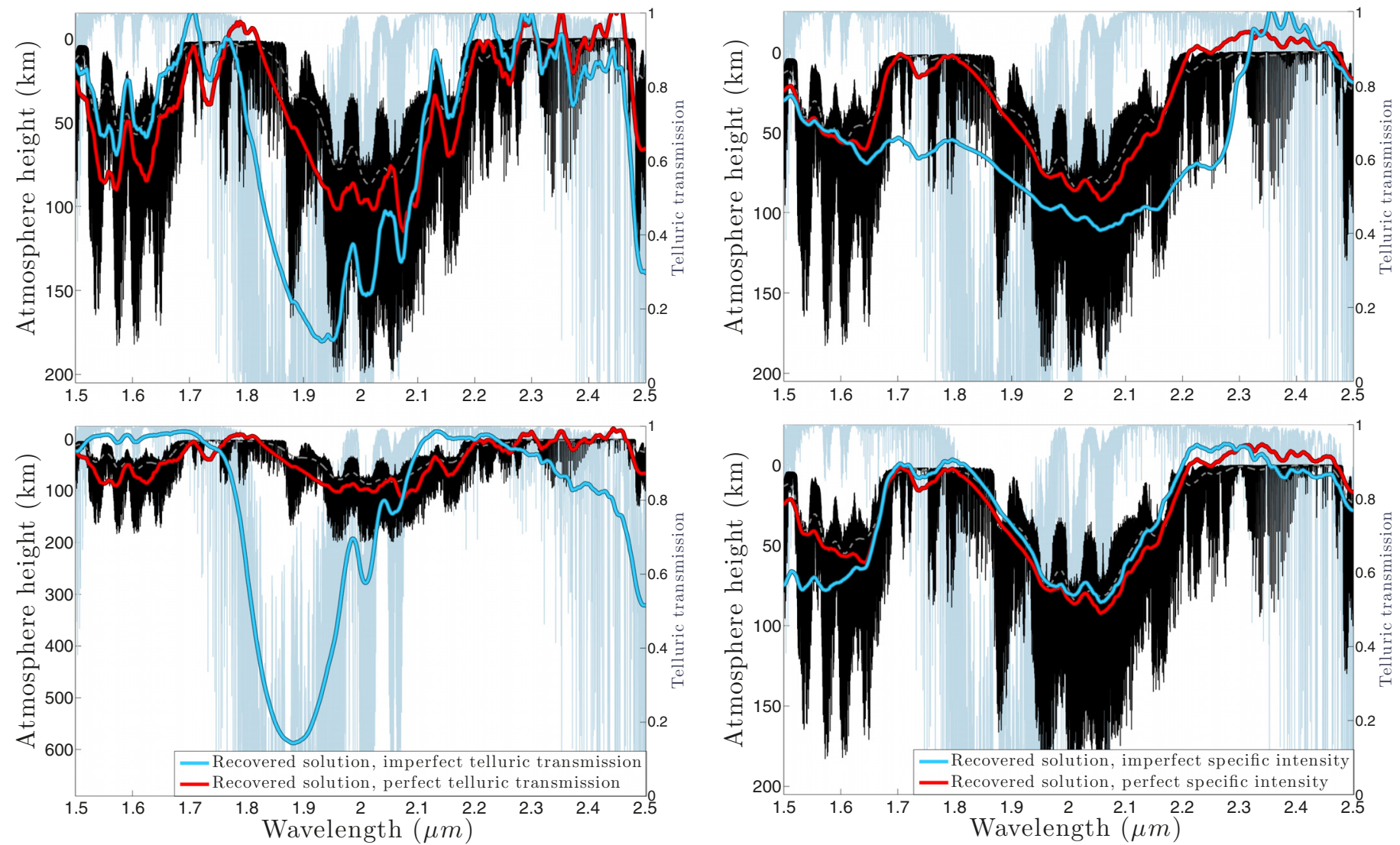

Fig. 7. Recovered exoplanetary transmission spectra from simulated transit observations of a hot super-Earth transiting M5 star. Here we test the effects of using imperfect telluric transmittance in the data analysis. Upper panel: random over/underestimation up to $\pm 2 \%$ of telluric transmittance. Lower panel: systematic underestimation up to $\pm 2 \%$ of telluric transmittance. Colour coding is the same as in Fig. 4.

rest frame of the exoplanet. By using synthetic telluric spectra, we do not need to spend any of the limited observing time during the transit to observe telluric spectrum template, but can instead spend all available time to observe the transiting exoplanet. Observations aimed at measuring the telluric atmosphere, by observing telluric standard stars or similar objects with known spectral features along the same line of sight as the transiting exoplanet, could still be carried out before and after the transit in order to get the starting and ending conditions of the atmosphere.

The accuracy of the telluric spectra must be high; systematic errors such as constant over- or under-estimation of telluric features manifests as spurious features in the solution. To investigate the needed level of accuracy, we tested cases with imperfect telluric spectra when applying the data-analysis method. First we used small non-systematic errors by alternating between over- and under-estimation of the telluric transmittance by up to $\pm 2 \%$. We still manage to recover the same major molecular absorption bands as in the case-perfect telluric transmittance, but artificial features were producedt in regions with strong telluric absorption. The effects of systematic errors were also examined, by always underestimating the telluric transmittance by up to $2 \%$ when performing the data analysis. In this case, the reconstruction is completely dominated by the errors in telluric transmittance, and the contribution from the exoplanet is no longer visible. Both tests are shown in Fig. 7.

The flux spectrum of the host star is needed for the inversion. This can be measured before or after the transit. Its

Fig. 8. Recovered exoplanetary transmission spectra from simulated transit observations of a hot super-Earth transiting M5 star. Here we test the effects of using imperfect stellar specific intensity spectra in the data analysis. Upper panel: specific intensity from quadratic limb darkening equations. Lower panel: specific intensity from model atmosphere with $5 \%$ too high effective temperature. Colour coding is the same as in Fig. 4.

stability or variability can be monitored outside the transit. Specific intensity spectra is more challenging. One approach is to use synthetic spectra based on a custom-made model of stellar atmosphere. The model can be validated by comparing the flux spectrum with observations outside transit. Limb darkening dependence is hard to verify. We examine the effects of errors in specific intensity by using imperfect specific intensity spectra in the data analysis. We use quadratic limb darkening equation in one test and modelled specific intensities with effective temperature 5\% higher than for the synthesis of observations. Results can be seen in Fig. 8. In both cases, the major absorption bands from the exoplanet are still recovered, but with limb darkening equations, errors in the shape of the continuum are introduced, while with imperfect model spectra, we only observe minor deviations from perfect intensity spectra. It is worth considering that the case with limb darkening equations is close to optimal, while the case with model spectra introduces deliberate errors. Model spectra still produce better results, so we recommend the use of model spectra over limb darkening equations.

Approximating the area-integrated specific intensity $\left(i_{\mathrm{p}}\right.$ and $i_{\mathrm{a}}$ in Eqs. (1)) with the value at the centre of the planet or using incorrect $\mu$-values will produce effects similar to the test above. In the cases presented here (hot-Jupiter in front of Solarsize star and super-Earth in front of $\mathrm{M}$ dwarf), the errors in specific intensity spectra will be less than $1 \%$, such small errors will not affect the end results in any meaningful way. 
Inhomogeneities on the stellar surface, such as starspots, can potentially also cause artificial features in the recovered solution. If the transiting exoplanet crosses one or several starspots, the light passing through the atmosphere will be significantly different from the assumed specific intensity used in the data analysis. The effects this has on the recovered exoplanet spectrum depends on the amount of exposures that are contaminated and on the fraction of the exoatmosphere that covers the spots. For young active stars, a significant fraction of the stellar surface can be covered by starspots. Transit spectroscopy will be very complicated for these stars. For solar-like stars, the amount of contamination from starspots will very likely be insignificant. For the small fully convective stars, starspot patterns are largely unknown and could cause problems depending of the size of individual spots and the fraction of the disk that is covered.

We performed a simple test where we simulate observations of a transiting hot-Jupiter crossing a solar-like star, where we replace some of the s pecific intensity spectra with the spectra of sunspots (data from Pierce Solar Telescope). We then ignore the spots when applying the data analysis method. For this test we used spots of sizes comparable to the planet, and a rather extreme case where the planet covers spots in $20 \%$ of the exposures ( $>50 \%$ of planet covered in $10 \%$ of exposures). Results from this test can be seen in Fig. 9. We managed to recover the same spectral features as when using a starspot free case. We therefore do not expect any major issues concerning starspots for solar-like stars. Ideally exposures contaminated by contribution from starspots should be removed from the data. The photometry of the transits can detect the starspots if the spots are large enough to produce a detectable brightness increase in the transit light curve as the planet passes over it. This spot detection has successfully been demonstrated by Pont et al. (2007), Carter et al. (2011), Sing et al. (2011).

Photometric measurements taken simultaneous with the spectroscopic measurements could help find the most problematic spots so that they can be removed from the data, at the same time giving the exact start and end of the transit. Unocculted starspots and other forms of stellar variability during the transit could potentially also cause problems, mainly in the form of errors in size determination of the solid part of the exoplanet. In Sect. 4.3 we explore the effects of errors in planetary radius. Stars covered by a large number of star spots, enough to alter the flux significantly, could also be problematic. In such cases the stellar spectrum underneath the planet will be different from the stellar flux, which would make synthetic specific intensity spectra less accurate if using observed stellar flux to validate the stellar model. For stars with solar-like spot coverage (less than $0.5 \%$ of the sun is covered by spots during solar maximum), this effect would be insignificant.

\subsection{Target selection and planet parameters}

The data analysis method requires the size of the planet to be known. This can be measured in the photometric transit light curve. Errors and uncertainties in the size of the planet will have two effects. First, this will shift the recovered spectrum along the atmospheric height axis, down for over-estimations and up for under-estimations. This effect is easily minimized by setting the continuum of the solution to zero. The second effect of errors in the size of the planet is the expected brightness decrease in the star as the planet moves in front of the stellar disk (characterized $v$ in Eq. (4)). Errors in this can leave an imprint of stellar or telluric spectra in the recovered solution. This can be solved by using our proposed way of calculating $v$ (Eq. (5)), which corrects

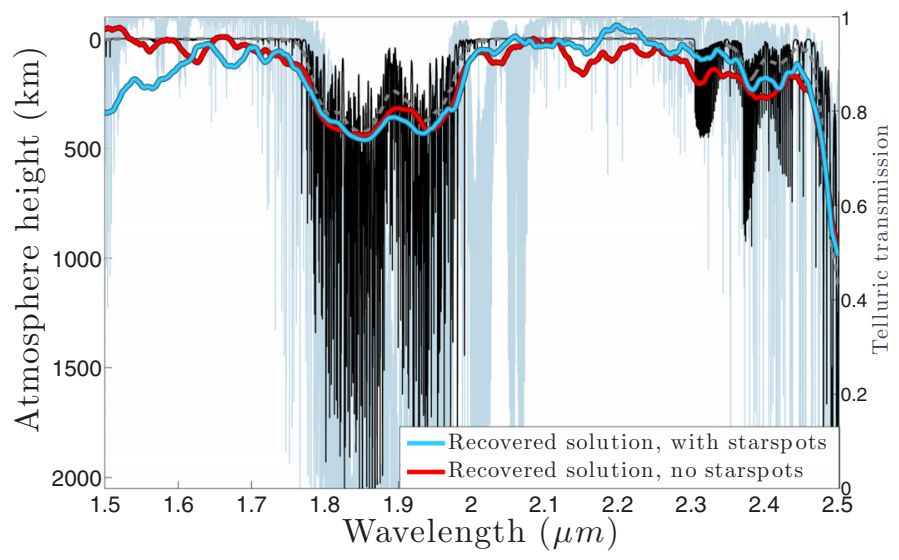

Fig. 9. Recovered exoplanetary transmission spectra from simulated transit observations of a hot-Jupiter transiting active G2 star. Here the planet crosses starspots during observations, this is then ignored in the data analysis. Colour coding is the same as in Fig. 4.

for errors in planetary size. Reasonably small errors in planetary size will only have a weak effect on the relative strength of absorption features. We show this by simulating two cases, one with $10 \%$ overestimation and one with $10 \%$ underestimation of planetary radius, which are the results after setting continuum to zero shown in Fig. 10.

Another potential problem is the case where there is no atmosphere (or a completely transparent or opaque atmosphere in all measured wavelengths). We investigated the ability of the dataanalysis method to distinguish between a planet with a semitransparent atmosphere and a planet with no atmosphere at all by simulation observations of the two cases, and then applied the data analysis to both cases in exactly the same way. Results are shown in Fig. 11. In the case with no atmosphere, the deviations from a flat continuum at height 0 are mainly due to the inverse method trying to fit the noise. In this case we cannot distinguish a planet with no atmosphere from a planet with a thin atmosphere (eclipsing height less than $100 \mathrm{~km}$ ). This upper limit can be reduced with less noisy observations (or observations from several transits). An atmosphere dominated by Rayleigh scattering in the observed wavelengths is similar to the case with no atmosphere. The needed level of precision to measure the small changes across the wavelengths range due to Rayleigh scattering is difficult to achieve with ground-based telescopes and could easily be mistaken for a planet with no atmosphere using this method. Here other observational techniques would be more appropriate, such as low-resolution space-based spectroscopy.

Planetary systems come in many different sizes and brightnesses. The relative projected area of the planet's semitransparent atmosphere to the area of the stellar disk is the most important factor for the required $\mathrm{S} / \mathrm{N}$ for transit spectroscopy. This can differ by a factor of 10000 between the most extreme cases. We applied the method to simulations of a multitude of different size ratios. To make it easier to account for different transit durations and observations during several transits, this was converted into $\mathrm{S} / \mathrm{N}$ per resolution element of all exposures combined (see Table 3). This is for the idealized case and that eclipsing height of the atmosphere strongly affects the required $\mathrm{S} / \mathrm{N}$.

The constantly growing body of known exoplanets includes many suitable candidates for transit spectroscopy of this type. In Table 4 we list some the most advantages exoplanets for observations of them. Data was taken from the Exoplanet Orbit Database (Wright et al. 2011). 
Table 3. Required $\mathrm{S} / \mathrm{N}$ per resolution element $(R=100000)$, all exposures combined.

\begin{tabular}{lllll}
\hline \hline Star & Hot-Jupiter $\left(1.0 R_{\text {Jup }}\right)$ & Hot-Neptune $\left(1.0 R_{\text {Nep }}\right)$ & Venus-like super-Earth $\left(2.5 R_{\oplus}\right)$ & Earth-twin $\left(1.0 R_{\oplus}\right)$ \\
\hline G2-star $\left(1.0 R_{\odot}\right)$ & $S / N>400$ & $S / N>3500$ & $S / N>20000$ & $S / N>150000$ \\
K2-star $\left(0.8 R_{\odot}\right)$ & $S / N>350$ & $S / N>2500$ & $S / N>12000$ & $S / N>90000$ \\
K8-star $\left(0.5 R_{\odot}\right)$ & $S / N>100$ & $S / N>1000$ & $S / N>5000$ & $S / N>36000$ \\
M5-star $\left(0.2 R_{\odot}\right)$ & $S / N>60$ & $S / N>200$ & $S / N>800$ & $S / N>5700$ \\
\hline
\end{tabular}
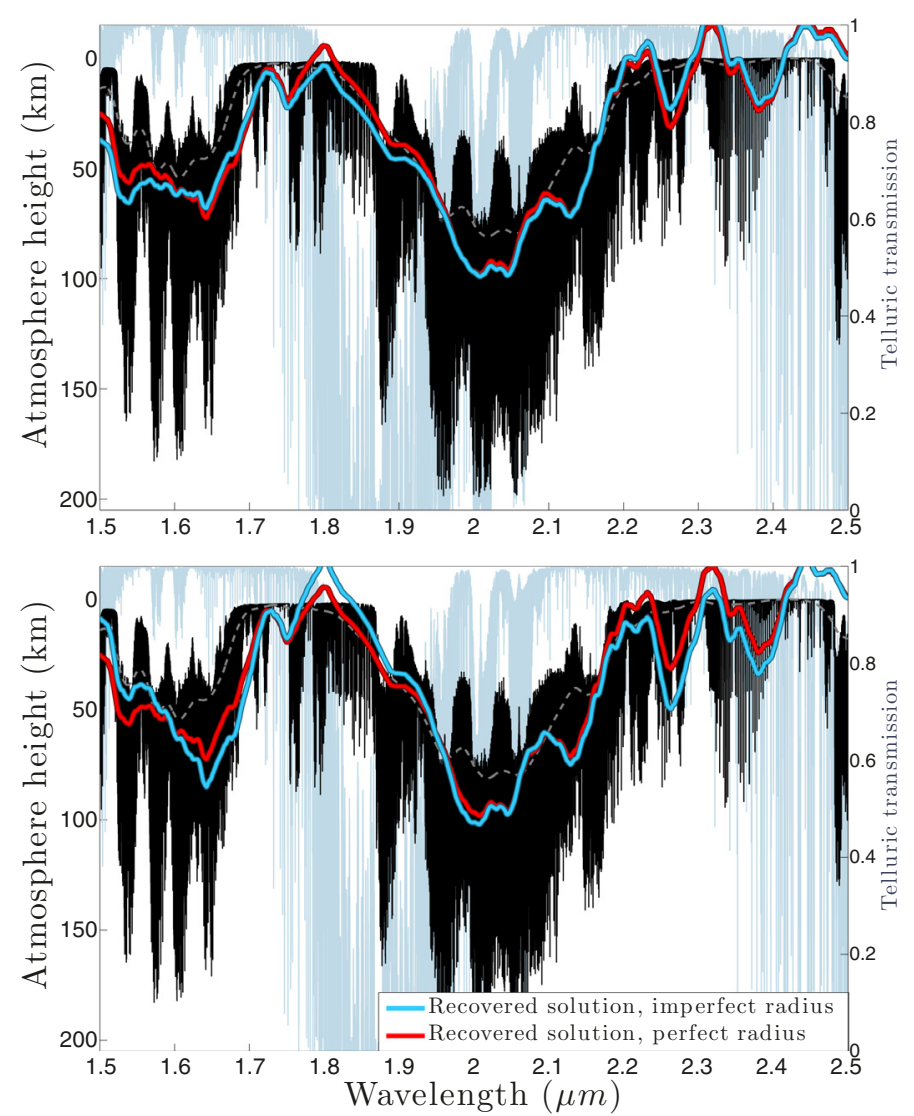

Fig. 10. Recovered exoplanetary transmission spectra from simulated transit observations of hot super-Earth transiting M5 star. Here we test the effects of using errors in estimated planetary radius in the data analysis. Upper panel: $10 \%$ over-estimation of planetary radius. Lower panel: $10 \%$ under-estimation of planetary radius. Colour coding is the same as in Fig. 4.

\section{Instruments}

High-resolution spectroscopy of exoplanetary atmospheres put stringent requirements on the instruments used for observations. We look into current, upcoming, and planned instruments that will be able to provide observations with sufficient $\mathrm{S} / \mathrm{N}$ and spectral resolution. Most of the current high-resolution NIR spectrographs suffer from short wavelength coverage, which do not have high enough resolution or a large enough photon-collecting area. The otherwise impressive echelle spectrograph CRIRES at VLT ( $R=100000,8 \mathrm{~m}$ primary) has a very narrow wavelength coverage and does not include a cross-dispersed mode to increase this, which makes the instrument hard to use with the method presented here. Other spectrographs with sufficient spectral coverage usually have spectral resolution well under 10000 , making observations with this method mostly pointless. There are, however, a few current instruments just at the lower edge of the needed requirements and several upcoming instruments that will

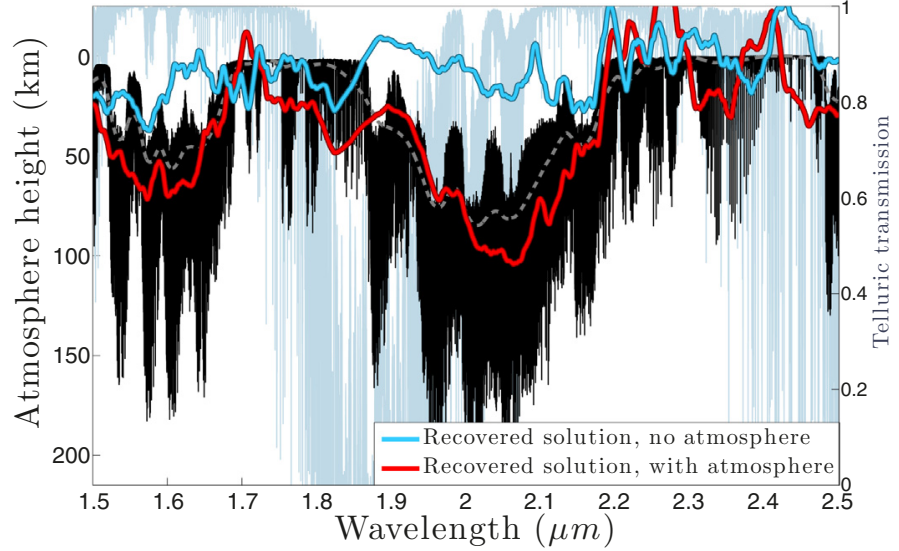

Fig. 11. Recovered exoplanetary transmission spectra from simulated transit observations of super-Earth transiting M5 star. In one case the exoplanet has an atmosphere, and in the other the exoplanet has no atmosphere, so here we show that this difference can be detected. Colour coding is the same as in Fig. 4.

Table 4. Currently known most suitable observational targets for the observational and data analysis method presented in this paper.

\begin{tabular}{lllc}
\hline \hline Exoplanet & $R_{\text {planet }}$ & $R_{\text {star }}$ & 2MASS $K_{\mathrm{S}}$ \\
\hline Hot-Jupiters & & & \\
HD 209458 b & $1.4 R_{\text {Jup }}$ & $1.2 R_{\odot}$ & 6.3 \\
HD 189733 b & $1.1 R_{\text {Jup }}$ & $0.76 R_{\odot}$ & 5.5 \\
HD 80606 b & $1.0 R_{\text {Jup }}$ & $1.0 R_{\odot}$ & 7.3 \\
WASP-80 b & $0.95 R_{\text {Jup }}$ & $0.57 R_{\odot}$ & 8.4 \\
Hot-Neptunes & & & \\
HAT-P-26 b & $1.6 R_{\text {Nep }}$ & $0.79 R_{\odot}$ & 9.6 \\
HAT-P-11 b & $1.2 R_{\text {Nep }}$ & $0.75 R_{\odot}$ & 7.0 \\
GJ 436 b & $1.1 R_{\text {Nep }}$ & $0.46 R_{\odot}$ & 6.1 \\
GJ 3470 b & $1.1 R_{\text {Nep }}$ & $0.50 R_{\odot}$ & 8.0 \\
Super-Earths & & & \\
GJ 1214 b & $2.6 R_{\oplus}$ & $0.21 R_{\odot}$ & 8.8 \\
Kepler-49 b & $2.7 R_{\oplus}$ & $0.53 R_{\odot}$ & 12.4 \\
Kepler-125 b & $2.3 R_{\oplus}$ & $0.51 R_{\odot}$ & 11.7 \\
HD 97658 b & $2.3 R_{\oplus}$ & $0.70 R_{\odot}$ & 5.7 \\
55 cnc e & $2.0 R_{\oplus}$ & $0.94 R_{\odot}$ & 4.0 \\
Earth-size planets & & & \\
Kepler-205 c & $1.6 R_{\oplus}$ & $0.55 R_{\odot}$ & 10.8 \\
Kepler-186 d & $1.4 R_{\oplus}$ & $0.47 R_{\odot}$ & 11.6 \\
Kepler-42 b & $0.76 R_{\oplus}$ & $0.17 R_{\odot}$ & 11.5 \\
Kepler-42 c & $0.71 R_{\oplus}$ & $0.17 R_{\odot}$ & 11.5 \\
\hline
\end{tabular}

be able to deliver observations with sufficient quality within the coming years.

The two best-suited current spectrographs are NIRSPEC (McLean et al. 1998) at Keck II and IRCS (Tokunaga et al. 1998) at the Subaru Telescope. NIRSPEC has a spectral resolution of 25000 and a simultaneous wavelength coverage of about about $0.2 \mu \mathrm{m}$. IRCS has similar capabilities, a slightly lower spectral resolution of 20000 , but the simultaneous wavelength coverage is better, around $0.4 \mu \mathrm{m}$ at the relevant wavelengths. 
These instruments are at the lower edge of the instrumental requirements, and characterization of gas giants transiting the brightest stars should be possible with these instruments. We show this by simulating observations from one transit of the hotJupiters HD 189733 b and HD 209458 b (see Table 4), using an instrument with spectral resolving power $R=20000$ at an $8 \mathrm{~m}$ telescope. Results shown in Fig. 12.

While these current instruments should be able to characterize the large planets transiting the brightest stars, reaching superEarths will not be possible unless large amounts of observing time is dedicated to this task. Driven largely by the need for precise radial velocity measurements of cool dwarf stars to detect Earth-size exoplanets, a number of upcoming high-resolution NIR spectrographs are currently planned. Many of these will be located at $4 \mathrm{~m}$ class telescopes, which will have a hard time reaching the needed $\mathrm{S} / \mathrm{N}$ for transit spectroscopy. A few of the proposed or in-progress instruments will, however, be located at bigger telescopes.

One such instrument is the HZPF (Mahadevan et al. 2010), a proposed NIR high-resolution spectrograph, which will have a spectral resolution of 80000 , a wavelength coverage of $0.9-1.7 \mu \mathrm{m}$ in a single exposure, and the photon collecting power of the Hobby-Eberly Telescope's (HET) primary $9 \mathrm{~m}$ mirror. At first glance, this instrument seems very promising, however, HET cannot be pointed towards any part of the sky, and one must instead wait for a star with a transiting planet to pass over the telescope at exactly the right time. This keeps HET from being well-suited to observing rare and time-limited phenomena like exoplanetary transits. Combing observations from several transits would also be very hard, which makes recovering exoplanetary contributions from all but the largest planets impossible.

The Subaru Telescope already has a NIR spectrograph capable of characterizing the most favourable cases (IRCS). There are, however, plans to add new high-resolution options to this telescope. IRCS-HRU (Terada et al. 2008) will increase the maximum possible resolution of IRCS up to 70000 in wavelengths longer than $1.4 \mu \mathrm{m}$. Another instrument, specialized in NIR radial velocity measurements is the IRD (Tamura et al. 2012), which will have a resolution of 70000 and a simultaneous spectral coverage of $1.2-1.85 \mu \mathrm{m}$. Finally there is WINERED (Yasui et al. 2008) with a resolution of 100000 and simultaneous spectral coverage $0.9-1.35 \mu \mathrm{m}$. All of these instrument have the needed requirements for exoplanetary transit spectroscopy with the method presented here, and they might even be able to reach some of the more favourable super-Earths if enough observing time has been granted.

The current high-resolution NIR spectrograph at VLT, CRIRES, suffers from a very limited spectral coverage in a single exposure $(0.05 \mu \mathrm{m})$. There is a proposed upgrade for this instrument, CRIRES+ (Oliva et al. 2012). This instrument will have the same spectral resolution (100000) and a significantly increased spectral coverage owing to the addition of a cross-dispersed mode. With the large photon collecting power of VLT's $8 \mathrm{~m}$ primary mirror and covering most of the $Y, J, H, K$, or $M$-bands in a single exposure, observations from this instrument will be able to characterize many giant planets transiting bright stars. One should also be able to reach super-Earths transiting the very small stars if combing several transits. Advantageous super-Earths for these types of observations are, however, hard to find within reach of Paranal observatory (mainly due to all Kepler objects being located too far north), and currently the only known super-Earth where

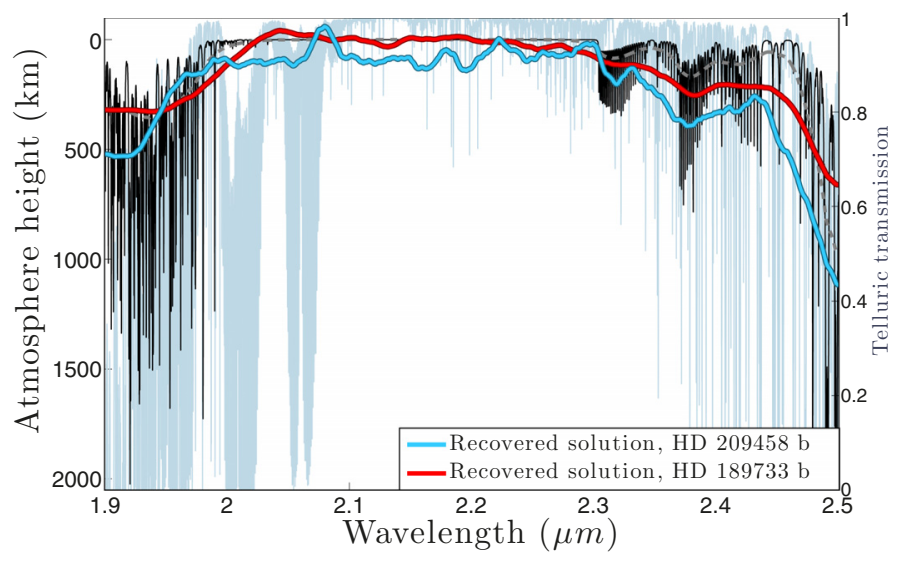

Fig. 12. Recovered exoplanetary transmission spectra from simulated transit observations of systems with similar parameters to the two most promising observational candidates (see Table 4), using NIR spectrograph IRCS at the Subaru Telescope. Colour coding is the same as in Fig. 4.

CRIRES+ observations will be sufficient for reproducing the transmission spectrum reliably is GJ $1214 \mathrm{~b}$.

The NIR spectrographs for the next generation of $30 \mathrm{~m}$ class telescopes are even more impressive. For the $39 \mathrm{~m}$ European Extremely Large Telescope (E-ELT), the current idea is HIRES; a combined optical and NIR spectrograph with resolving power of at least 100000 and simultaneous wavelength coverage of 1.0-2.7 $\mu \mathrm{m}$. With the large photon collecting area of a $39 \mathrm{~m}$ mirror, observations during a single transits corresponds to observing more than 20 successive transits with an $8 \mathrm{~m}$ telescope. This makes characterizations of exoplanets orbiting faint stars feasible, as well as recovering high-resolution spectra from large planets around bright stars. (Results could be similar to Fig. 6.) The transit spectroscopy of Earth-size planets orbiting solar-like stars would still be hard because of the extremely small fraction of the stellar disk that is covered by the planetary atmosphere. To show the power of the photon collecting area of E-ELT, combined with a high-resolution spectrograph such as HIRES, we simulated observations of a single transit of GJ 1214 b (see Table 4, assuming a atmospheric height of $150 \mathrm{~km}$ ). This small planet orbits a faint star and would require observations of several transits with smaller telescopes to reach sufficient $\mathrm{S} / \mathrm{N}$, but with a $39 \mathrm{~m}$ mirror one would able to detect the main absorption bands from observations during a single transit. Results from this test are shown in Fig. 13, including comparison with observations with a $8 \mathrm{~m}$ telescope.

The Thirty Meter Telescope (TMT) also has planned a highresolution NIR spectrograph, NIRES. This echelle spectrograph will be able to achieve spectral resolution up to 100000 and will operate in 1-5 $\mu \mathrm{m}$. A cross-dispersed mode will enable observations from 1.0-2.5 $\mu \mathrm{m}$ simultaneously; as a result, apart from a smaller photon collecting area, the performance of NIRES will be very similar to E-ELT's HIRES. However, since the TMT is planned to be built on Mauna Kea, the telescopes will have access to the northern sky, where currently the majority of the favourable super-Earths and Earth-size exoplanets for transit spectroscopy are located.

\section{Observations}

To test the reduction procedure on real data we applied the data analysis method to CRIRES observations of HD 209458 b 


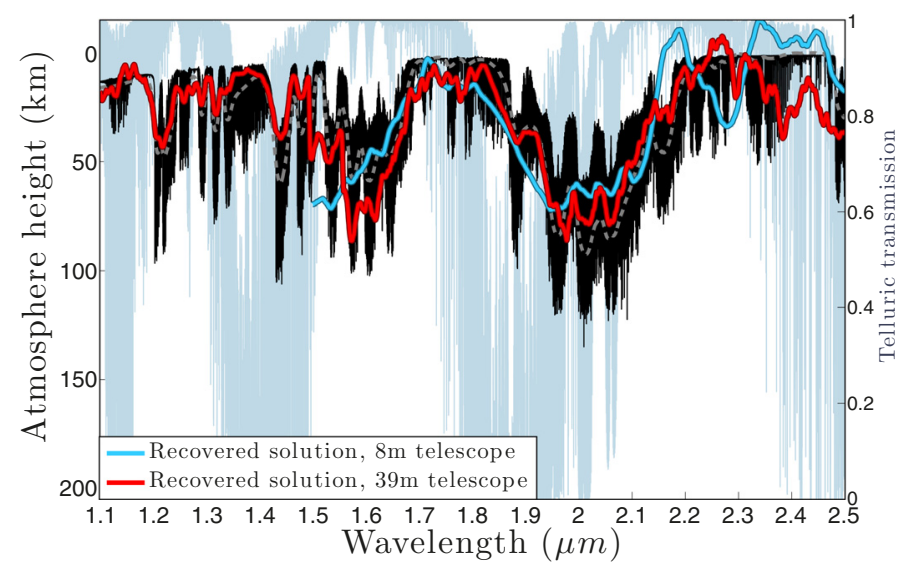

Fig. 13. Recovered exoplanetary transmission spectra from simulated transit observations of a system with similar parameters GJ 1214 b (see Table 4). Comparison between a $39 \mathrm{~m}$ and $8 \mathrm{~m}$ telescope. Colour coding is the same as in Fig. 4.

(Snellen et al. 2010). Data consists of 51 spectra (30 during transit) of a single three-hour transit of HD 209458 b (see Table 4), targeting a CO-band around 2.30 to $2.35 \mu \mathrm{m}$. We only used data from Detectors 2 and 3 owing to poor data quality in Detectors 1 and 4 . In this region, there are few stellar lines, which reduces the need for accurate specific intensity spectra. Instead we used quadratic limb darkening law to model changes in stellar intensity as function of limb distance. Flux spectra are taken from the out-of-transit observations. Model telluric spectra were created using similar procedure to those described by Snellen et al. (2010), except that we do not assign zero weight to the pixels in which we expect exoplanetary signal; instead, we increase the weight of pixels during out-of-transit exposures.

Owing to the short wavelength range, we cannot use the optimal regularization parameter based on data quality and expected signal strength, because doing so would result in a flat featureless solution. Instead we decrease regularization to what would be appropriate for recovering individual absorption lines in spite of the data not having the needed quality to recover individual lines. The recovered solution is shown in Fig. 14, along with the placement of the CO lines previously detected in the same data set. The solution appears noisy, and only a few individual CO lines seem to be reconstructed. From this data it is not possible to directly identify the absorbers in the atmosphere of HD 209458 b. However, analysis of multiple CO lines will increase the signal. Stacking the available CO lines (ignoring the lines at 2317.7 and $2323.6 \mathrm{~nm}$ due to several bad pixels close to line cores) and normalizing by the average value in the wings reveals an overall absorption feature centred on the core of the combined CO lines (see inserted panel in Fig. 14). To show that the recovered feature is not a coincidence, we also stack and normalize the recovered solution over randomized line positions. From 100000 different realizations of such randomized line positions, only $1 \%$ shows absorption at the core of the line stronger than what we get from combing positions of CO lines.

\section{Conclusions}

The data analysis method presented here makes the recovery of exoplanetary atmospheric transmittance spectrum using highresolution observations of transiting exoplanets robust. There are several advantages to this method over the classical narrow band filter photometry approach. One of the most important aspects is

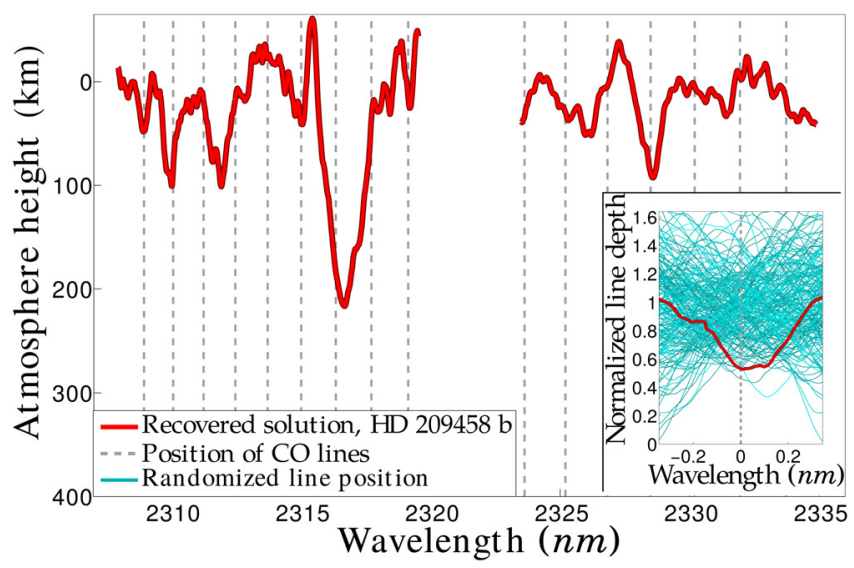

Fig. 14. Recovered exoplanetary transmission spectrum of HD 209458 b from CRIRES observations of a single transit. The expected positions of CO lines are shown as dashed grey lines. Only a few of the $16 \mathrm{CO}$ lines in the observed wavelength range are visible in the recovered spectrum. Inserted panel: stacked exoplanet spectra (normalized to average value in wings) over the expected position of CO lines (shown in red) and small sample (200) of 100000 realizations of randomized positions of line centres (shown in turquoise). Only $1 \%$ of the randomized line positions show stronger line depth than the CO lines.

the interpretation of the data, which becomes more straightforward thanks to higher spectral resolution and a wide wavelength coverage. Given the estimates of the physical conditions in the planetary atmosphere (pressure, temperature regime, UV-flux, etc.), a first approximation of chemical composition could be made directly from the recovered spectrum, without much need for atmospheric models.

Molecular species responsible for strong absorption features (such as $\mathrm{H}_{2} \mathrm{O}, \mathrm{CO}_{2}, \mathrm{CO}, \mathrm{CH}_{4}$ ) could be identified from the shape, placement, line spacing, and relative strength of recovered absorption bands. With broad enough spectral coverage to detect multiple absorption bands from a single molecular species, false detections could be minimized, and they would enable us to distinguish between species with overlapping absorption bands. From such a first approximation of the chemical composition, the next step would be to create a grid of planetary models with a range of relative abundances and temperature and pressure structures. Finding the model with the best fit to observations could now be made with fewer uncertainties, mainly due to the first approximation of chemical composition already made, but also due to the large number of data points that come with good spectral resolution and wide wavelength coverage.

This method requires a special set of observations. To optimize observations, many short exposures (around 1-2 min) of the star are needed while the planet transits. Along with this, accurate stellar flux spectrum (without transiting planet), specific intensities and telluric transmittance, corresponding to each individual exposure, are all needed. In order not to reduce the observing time of the actual target, which is limited by the transit duration, we propose using synthetic telluric spectra fitted to observations. We estimate that the requirements of instruments and observational targets are able to produce observations with sufficient quality. Instruments need high spectral resolution (>20000), good simultaneous spectral coverage $(>0.1 \mu \mathrm{m})$, and a large photon collecting area $(>8 \mathrm{~m})$.

We have identified potentially problematic issues. Large errors in telluric transmission spectra and stellar specific intensity can present as artificial features in the recovered exoplanet 
spectrum. We propose ways to minimize these problems and show that even under non-optimal conditions recovering the exoplanet spectrum is still possible.

For target selection, we defined the important aspects of targets and produced a short list of the most advantageous starplanet systems known to date (November 2014). The critical property of a target when performing transit spectroscopy is the relative size of the planet to its star. This makes the atmospheres of large gas giants generally much easier to recover. For spectral characterization of Jupiter-size exoplanets, this data analysis method using observations with high-resolution spectrometers at $8 \mathrm{~m}$ class telescopes seem promising. Recovering transmission spectra with this method will be possible once enough instrumentshave been constructed for most known gas giants orbiting bright stars. The chemical composition of these worlds might give us an insight into their origins.

Smaller planets, super-Earths, and Earth-size planets are more difficult to characterize. We expect to be able to distinguish spectral features originating in their atmospheres given sufficient observing time, accurate stellar models, and advantageous properties of the host star (small and bright). Unfortunately, very few favourable candidates are known, and most are located out of the reach of two of the most promising upcoming instruments, CRIRES+ at VLT and HIRES at E-ELT. However, the success of the Kepler space missions has demonstrated that the chances of finding observationally advantageous super-Earths on the southern hemisphere is high, so all we need are dedicated space-based transit surv eys.

Acknowledgements. This research made use of the Exoplanet Orbit Database and the Exoplanet Data Explorer at exoplanets.org. NSO/Kitt Peak FTS data used here were produced by NSF/NOAO.

\section{References}

Ballester, G. E., Sing, D. K., \& Herbert, F. 2007, Nature, 445, 511 Bean, J. L., Miller-Ricci Kempton, E., \& Homeier, D. 2010, Nature, 468, 669 Bean, J. L., Désert, J.-M., Kabath, P., et al. 2011, ApJ, 743, 92 Berta, Z. K., Charbonneau, D., Désert, J.-M., et al. 2012, ApJ, 747, 35 Boss, A. P. 1997, Science, 276, 1836

Brown, T. M. 2001, ApJ, 553, 1006

Cáceres, C., Kabath, P., Hoyer, S., et al. 2014, A\&A, 565, A7

Carter, J. A., Winn, J. N., Holman, M. J., et al. 2011, ApJ, 730, 82

Castan, T., \& Menou, K. 2011, ApJ, 743, L36

Charbonneau, D., Brown, T. M., Latham, D. W., \& Mayor, M. 2000, ApJ, 529, L45

Charbonneau, D., Brown, T. M., Noyes, R. W., \& Gilliland, R. L. 2002, ApJ, 568,377

Coleman, G. A. L., \& Nelson, R. P. 2014, MNRAS, 445, 479

Colón, K. D., \& Gaidos, E. 2013, ApJ, 776, 49
Croll, B., Albert, L., Jayawardhana, R., et al. 2011, ApJ, 736, 78

Crossfield, I. J. M., Barman, T., \& Hansen, B. M. S. 2011, ApJ, 736, 132 de Mooij, E. J. W., Brogi, M., de Kok, R. J., et al. 2012, A\&A, 538, A46 de Mooij, E. J. W., Brogi, M., de Kok, R. J., et al. 2013, ApJ, 771, 109

Désert, J.-M., Bean, J., Miller-Ricci Kempton, E., et al. 2011, ApJ, 731, L40

Fraine, J. D., Deming, D., Gillon, M., et al. 2013, ApJ, 765, 127

Gordley, L. L., Marshall, B. T., \& Chu, D. A. 1994, J. Quant. Spectr. Rad. Transf., 52, 563

Gustafsson, B., Edvardsson, B., Eriksson, K., et al. 2008, A\&A, 486, 951

Hubbard, W. B., Fortney, J. J., Lunine, J. I., et al. 2001, ApJ, 560, 413

Jha, S., Charbonneau, D., Garnavich, P. M., et al. 2000, ApJ, 540, L45

Kausch, W., Noll, S., Smette, A., et al. 2014, in Astronomical Data Anaylsis Softward and Systems XXIII, eds. N. Manset, \& P. Forshay, ASP Conf. Ser., 485,403

Knutson, H. A., Benneke, B., Deming, D., \& Homeier, D. 2014, Nature, 505, 66 Kreidberg, L., Bean, J. L., Désert, J.-M., et al. 2014, Nature, 505, 69

Lecavelier Des Etangs, A., Vidal-Madjar, A., Désert, J.-M., \& Sing, D. 2008, A\&A, 485, 865

Léger, A., Selsis, F., Sotin, C., et al. 2004, Icarus, 169, 499

Mahadevan, S., Ramsey, L., Redman, S., et al. 2010, in Pathways Towards Habitable Planets, eds. V. Coudé du Foresto, D. M. Gelino, \& I. Ribas, ASP Conf. Ser., 430, 272

McLean, I. S., Becklin, E. E., Bendiksen, O., et al. 1998, in Infrared Astronomical Instrumentation, ed. A. M. Fowler, SPIE Conf. Ser., 3354, 566

Miguel, Y., \& Kaltenegger, L. 2014, ApJ, 780, 166

Murgas, F., Pallé, E., Cabrera-Lavers, A., et al. 2012, A\&A, 544, A41

Nagasawa, M., Ida, S., \& Bessho, T. 2008, ApJ, 678, 498

Narita, N., Fukui, A., Ikoma, M., et al. 2013, ApJ, 773, 144

Oliva, E., Hatzes, A., Piskunov, N., et al. 2012, in SPIE Conf. Ser., 8446

Pont, F., Gilliland, R. L., Moutou, C., et al. 2007, A\&A, 476, 1347

Pont, F., Gilliland, R. L., Knutson, H., Holman, M., \& Charbonneau, D. 2009, MNRAS, 393, L6

Redfield, S., Endl, M., Cochran, W. D., \& Koesterke, L. 2008, ApJ, 673, L87

Rogers, L. A., \& Seager, S. 2010, ApJ, 716, 1208

Rothman, L. S., Gordon, I. E., Barbe, A., et al. 2009, J. Quant. Spectr. Rad. Transf., 110, 533

Seager, S., \& Sasselov, D. D. 2000, ApJ, 537, 916

Seifahrt, A., Käufl, H. U., Zängl, G., et al. 2010, A\&A, 524, A11

Sing, D. K., Pont, F., Aigrain, S., et al. 2011, MNRAS, 416, 1443

Snellen, I. A. G., de Kok, R. J., de Mooij, E. J. W., \& Albrecht, S. 2010, Nature, 465, 1049

Swain, M. R., Vasisht, G., \& Tinetti, G. 2008, Nature, 452, 329

Tamura, M., Suto, H., Nishikawa, J., et al. 2012, in SPIE Conf. Ser., 8446

Terada, H., Yuji, I., Kobayashi, N., et al. 2008, in SPIE Conf. Ser., 7014

Teske, J. K., Turner, J. D., Mueller, M., \& Griffith, C. A. 2013, MNRAS, 431, 1669

Tokunaga, A. T., Kobayashi, N., Bell, J., et al. 1998, in Infrared Astronomical Instrumentation, ed. A. M. Fowler, SPIE Conf. Ser., 3354, 512

Vidal-Madjar, A., Lecavelier des Etangs, A., Désert, J.-M., et al. 2003, Nature, 422,143

Vidal-Madjar, A., Désert, J.-M., Lecavelier des Etangs, A., et al. 2004, ApJ, 604, L69

Vidal-Madjar, A., Huitson, C. M., Bourrier, V., et al. 2013, A\&A, 560, A54

Wilson, P. A., Colón, K. D., Sing, D. K., et al. 2014, MNRAS, 438, 2395

Winn, J. N. 2010, ArXiv e-prints [arXiv: 1001.2010]

Wright, J. T., Fakhouri, O., Marcy, G. W., et al. 2011, PASP, 123, 412

Yasui, C., Kondo, S., Ikeda, Y., et al. 2008, in SPIE Conf. Ser., 7014 